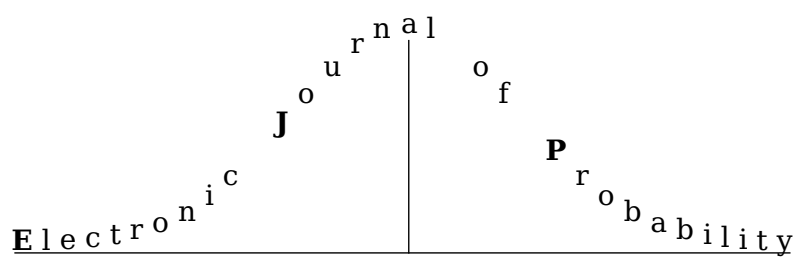

\title{
Free energy of directed polymers in random environment in $1+1$-dimension at high temperature*
}

\author{
Makoto Nakashima $^{\dagger}$
}

\begin{abstract}
We consider the free energy $F(\beta)$ of the directed polymers in random environment in $1+1$-dimension. It is known that $F(\beta)$ is of order $-\beta^{4}$ as $\beta \rightarrow 0[3,28,42]$. In this paper, we will prove that under a certain dimension free concentration condition on the potential,
\end{abstract}

$$
\lim _{\beta \rightarrow 0} \frac{F(\beta)}{\beta^{4}}=\lim _{T \rightarrow \infty} \frac{1}{T} P_{\mathcal{Z}}\left[\log \mathcal{Z}_{\sqrt{2}}(T)\right]=-\frac{1}{6}
$$

where $\left\{\mathcal{Z}_{\beta}(t, x): t \geq 0, x \in \mathbb{R}\right\}$ is the unique mild solution to the stochastic heat equation

$$
\frac{\partial}{\partial t} \mathcal{Z}=\frac{1}{2} \Delta \mathcal{Z}+\beta \mathcal{Z} \dot{\mathcal{W}}, \lim _{t \rightarrow 0} \mathcal{Z}(t, x) d x=\delta_{0}(d x)
$$

where $\mathcal{W}$ is a time-space white noise and

$$
\mathcal{Z}_{\beta}(t)=\int_{\mathbb{R}} \mathcal{Z}_{\beta}(t, x) d x
$$

Keywords: directed polymers; free energy; continuum directed polymer. AMS MSC 2010: Primary 82D60, Secondary 82C44.

Submitted to EJP on April 2, 2018, final version accepted on March 17, 2019.

In a general probability space $(\Omega, \mathcal{F}, P)$, we denote by $P[X]$ the expectation of random variable $X$ with respect to $P$. Let $\mathbb{N}_{0}=\{0,1,2, \cdots\}, \mathbb{N}=\{1,2,3, \cdots\}$, and $\mathbb{Z}=\{0, \pm 1, \pm 2, \cdots\}$. Let $C_{x_{1}, \cdots, x_{p}}$ or $C\left(x_{1}, \cdots, x_{p}\right)$ be a non-random constant which depends only on the parameters $x_{1}, \cdots, x_{p}$.

*Supported by JSPS Grant-in-Aid for Young Scientists (B) 26800051.

${ }^{\dagger}$ Graduate School of Mathematics, Nagoya University, Furocho, Chikusaku, Nagoya, Japan.

E-mail: nakamako@math.nagoya-u.ac.jp 


\section{Introduction and main result}

Directed polymers in random environment was introduced by Henly and Huse in the physical literature to study the influence by impurity of media to polymer chain [25]. In particular, random media is given as i.i.d. time-space random variables and the shape of polymer is achieved as time-space path of walk whose law is given by Gibbs measure with the inverse temperature $\beta \geq 0$, that is, time-space trajectory $s$ up to time $n$ appears as a realization of a polymer with probability

$$
\mu_{\beta, n}(s)=\frac{1}{Z_{\beta, n}} \exp \left(\beta H_{n}(s)\right) P_{S}^{0}\left(S_{[0, n]}=s\right), \quad s \in\left(\mathbb{Z}^{d}\right)^{n+1},
$$

where $H_{n}(s)$ is a Hamiltonian of the trajectory $s=\left(s_{0}, \cdots, s_{n}\right),\left(S, P_{S}^{x}\right)$ is the simple random walk on $\mathbb{Z}^{d}$ starting from $x \in \mathbb{Z}^{d}, S_{[0, n]}=\left(S_{0}, S_{1}, \cdots, S_{n}\right) \in\left(\mathbb{Z}^{d}\right)^{n+1}$, and $Z_{\beta, n}$ is the normalizing constant which is called the quenched partition function.

There exists $\beta_{1}$ such that if $\beta<\beta_{1}$, then the effect of random environment is weak and if $\beta>\beta_{1}$, then environment has a meaningful influence. This phase transition is characterized by the uniform integrability of the normalized partition functions. Also, we have another phase transition characterized by the non-triviality of the free energy, i.e. there exists $\beta_{2}$ such that if $\beta<\beta_{2}$, then the free energy is trivial and if $\beta>\beta_{2}$, then the free energy is non-trivial. The former phase transition is referred to weak versus strong disorder phase transition and the latter one is referred to strong versus very strong disorder phase transition. We have some known results on the phase transitions: $\beta_{1}=\beta_{2}=0$ when $d=1,2[21,28]$ and $\beta_{2} \geq \beta_{1}>0$ when $d \geq 3[13,19]$. In particular, the best lower bound of $\beta_{1}$ is obtained by Birkner et.al. by using size-biased directed polymers and random walk pinning model [7, 9, 11, 12, 10, 35].

There has been a lot of progress for $\mathbb{Z}^{d}$-lattice model in the past three decades $[13,15,19,20,16,21,28,6]$. Also, one dimensional case has received considerable attention in connection with KPZ equation [2, 1, 37]. In particular, it is believed that the critical exponent for the transversal fluctuation of the path is $\frac{2}{3}$ and the critical exponent for the longitudinal fluctuation of the free energy is $\frac{1}{3}$ for $\beta \neq 0$ [20]. This conjecture and several problems are partially solved under the integrable settings $[40,23,18]$.

Recent progress is reviewed in [17].

\subsection{Model and main result}

To define the model precisely, we introduce some random variables.

- (Random environment) Let $\left\{\eta(n, x):(n, x) \in \mathbb{N} \times \mathbb{Z}^{d}\right\}$ be $\mathbb{R}$-valued i.i.d.random variables with

$$
\begin{aligned}
& Q[\eta(n, x)]=0, Q\left[\eta(n, x)^{2}\right]=1, \text { and } \\
& \lambda(\beta)=\log Q[\exp (\beta \eta(n, x))] \in \mathbb{R} \text { for any } \beta \in \mathbb{R},
\end{aligned}
$$

where $Q$ is the law of $\eta^{\prime}$ s.

- (Simple random walk) Let $\left(S, P_{S}^{x}\right)$ be a simple random walk on $\mathbb{Z}^{d}$ starting from $x \in \mathbb{Z}^{d}$ which is independent of $\left\{\eta(n, x):(n, x) \in \mathbb{N} \times \mathbb{Z}^{d}\right\}$. We write $P_{S}=P_{S}^{0}$ for simplicity.

Then, the Hamiltonian $H_{n}(s)$ is given by

$$
H_{n}(s)=H_{n}(s, \eta)=\sum_{k=1}^{n} \eta\left(k, s_{k}\right), \quad s=\left(s_{0}, \cdots, s_{n}\right) \in\left(\mathbb{Z}^{d}\right)^{n+1}
$$


and

$$
Z_{\beta, n}=Z_{\beta, n}(\eta)=P_{S}\left[\exp \left(\beta \sum_{k=1}^{n} \eta\left(k, S_{k}\right)\right)\right]
$$

It is clear that

$$
Q\left[Z_{\beta, n}(\eta)\right]=\exp (n \lambda(\beta))
$$

for any $\beta \in \mathbb{R}$.

The normalized partition function is defined by

$$
\begin{aligned}
W_{\beta, n}(\eta) & :=\frac{Z_{\beta, n}(\eta)}{Q\left[Z_{\beta, n}(\eta)\right]} \\
& =Z_{\beta, n}(\eta) \exp (-n \lambda(\beta)) \\
& =P_{S}\left[\exp \left(\beta H_{n}(S)-n \lambda(\beta)\right)\right] \\
& =P_{S}\left[\prod_{k=1}^{n} \zeta_{k, S_{k}}(\beta, \eta)\right]
\end{aligned}
$$

where we write for each $(n, x) \in \mathbb{N} \times \mathbb{Z}^{d}$

$$
\zeta_{n, x}(\beta, \eta)=\exp (\beta \eta(n, x)-\lambda(\beta)) .
$$

Then, the following limit exists $Q$-a.s. and in $L^{1}(Q)[19,22]$ :

$$
\begin{aligned}
F(\beta) & =\lim _{N \rightarrow \infty} \frac{1}{N} \log W_{\beta, N}(\eta) \\
& =\lim _{N \rightarrow \infty} \frac{1}{N} Q\left[\log W_{\beta, N}(\eta)\right] \\
& =\sup _{N \geq 1} \frac{1}{N} Q\left[\log W_{\beta, N}(\eta)\right] .
\end{aligned}
$$

The fact that the limit in $N$ can be replaced by supremum in $N$ plays a key role in the proof of our main theorem.

The limit $F(\beta)$ is a non-random constant and called the quenched free energy. Jensen's inequality implies that

$$
F(\beta) \leq \lim _{N \rightarrow \infty} \frac{1}{N} \log Q\left[W_{\beta, N}(\eta)\right]=0 .
$$

It is known that $F(\beta)<0$ if $\beta \neq 0$ when $d=1,2[21,28]$ and $F(\beta)=0$ for sufficiently small $|\beta|$ when $d \geq 3$.

Recently, the asymptotics of $F(\beta)$ near high temperature $(\beta \rightarrow 0)$ are studied: There exists $\beta_{0}>0$ and $c \in(0, \infty)$ such that for $\beta \in\left(-\beta_{0}, \beta_{0}\right)$

$$
-c^{-1} \beta^{4} \leq F(\beta) \leq-c \beta^{4}, \text { if } d=1
$$

[28, Theorem 1.4, Theorem 1.5], [42, Theorem 1.1], [3, Theorem 1.3] and

$$
\lim _{\beta \rightarrow 0} \beta^{2} \log |F(\beta)|=-\pi, \text { if } d=2
$$

[28, Theorem 1.6], [34, Theorem 1.2], [6, Theorem 1.1].

In particular, it is conjectured $[38,39,17]$ that when $d=1$,

$$
\lim _{\beta \rightarrow 0} \frac{1}{\beta^{4}} F(\beta)=-\frac{1}{24},
$$

where $\frac{1}{24}$ appears in the literature of stochastic heat equation or KZP equation [8, 4].

Our main result answers this conjecture under a certain condition on $\eta$. 
Theorem 1.1. Suppose $d=1$. Also, we assume (1.1).

(i) (Upper estimate) We have that

$$
\varlimsup_{\beta \rightarrow 0} \frac{F(\beta)}{\beta^{4}} \leq-\frac{1}{6}
$$

(ii) (Sharp assymptotics) We consider the following dimension free concentration inequality condition on $\eta$ : There exists a non-decreasing function $g:(-\infty, 0) \rightarrow[0, \infty)$ such that

$$
\int_{-\infty}^{0} g(t) d t<\infty
$$

and that for any $m \in \mathbb{N}$ and for any differentiable convex function $f: \mathbb{R}^{m} \rightarrow \mathbb{R}$, we have that

$$
Q(f(\eta)<a-t) Q(f(\eta)>a,|\nabla f(\eta)| \leq c) \leq g\left(\frac{t}{c}\right), a \in \mathbb{R}, t, c \in(0, \infty)
$$

where $\eta=\left\{\eta_{1}, \cdots, \eta_{n}\right\}$ are i.i.d. random variables with the marginal law $Q\left(\eta_{e} \in d x\right)$ and $|\nabla f(\eta)|=\sqrt{\sum_{i=1}^{m}\left|\frac{\partial}{\partial \eta_{i}} f(\eta)\right|^{2}}$.

If in addition, we assume the above dimension free concentration inequality, then we have

$$
\lim _{\beta \rightarrow 0} \frac{1}{\beta^{4}} F(\beta)=-\frac{1}{6} .
$$

The following theorem by Alberts, Khanin and Quastel is a key theorem to obtain our main theorem.

Theorem 1.2. ([2, Theorem 2.1, Lemma A.1]) Suppose $d=1$. Let $\left\{\beta_{n}: n \geq 1\right\}$ be a $\mathbb{R}$-valued sequence with $\beta_{n} \rightarrow 0$ and $r>0$. Then, the sequence $\left\{W_{r \beta_{n},\left\lfloor T \beta_{n}^{-4}\right\rfloor}(\eta): n \geq 1\right\}$ is $L^{2}(Q)$-bounded and converges in distribution to $\mathcal{Z}_{r \sqrt{2}}(T)=\int_{\mathbb{R}} \mathcal{Z}_{r \sqrt{2}}(T, x) d x$ for each $T>0$, where $\mathcal{Z}_{\beta}(T)$ is the partition function of the continuum directed polymer for $\beta \geq 0$ at $T>0: \mathcal{Z}_{\beta}^{x}(t, y)$ is the unique mild solution to the stochastic heat equation

$$
\partial \mathcal{Z}=\frac{1}{2} \Delta \mathcal{Z}+\beta \mathcal{Z} \dot{\mathcal{W}}
$$

with the initial condition $\lim _{t \rightarrow 0} \mathcal{Z}(t, y) d y=\delta_{x}(d y)$ and $\mathcal{W}$ is a time-space white noise and $P_{\mathcal{Z}}$ is the law of $\mathcal{Z}_{\beta}^{x}$. We write

$$
\mathcal{Z}_{\beta}^{x}(t)=\int_{\mathbb{R}} \mathcal{Z}_{\beta}^{x}(t, y) d y
$$

and $\mathcal{Z}_{\beta}(t)=\mathcal{Z}_{\beta}^{0}(t)$ for simplicity.

Here, we will give an intuitive explanation of our main theorem. We have from (1.3) that for fixed $n \in \mathbb{N}$

$$
F\left(\beta_{n}\right)=\lim _{T \rightarrow \infty} \frac{1}{\left\lfloor T \beta_{n}^{-4}\right\rfloor} Q\left[\log W_{\beta_{n},\left\lfloor T \beta_{n}^{-4}\right\rfloor}(\eta)\right]
$$

Then,

$$
\lim _{n \rightarrow \infty} \frac{F\left(\beta_{n}\right)}{\beta_{n}^{4}}=\lim _{n \rightarrow \infty} \lim _{T \rightarrow \infty} \frac{1}{\beta_{n}^{4}\left\lfloor T \beta_{n}^{-4}\right\rfloor} Q\left[\log W_{\beta_{n},\left\lfloor T \beta_{n}^{-4}\right\rfloor}(\eta)\right] .
$$


Therefore, if we can exchange the limits in $n$ and $T$ and $\left\{\log W_{\beta_{n},\left\lfloor T \beta_{n}^{-4}\right\rfloor}(\eta): n \in \mathbb{N}\right\}$ is uniformly integrable, then we have that

$$
\begin{aligned}
\lim _{n \rightarrow \infty} \frac{F\left(\beta_{n}\right)}{\beta_{n}^{4}} & =\lim _{n \rightarrow \infty} \lim _{T \rightarrow \infty} \frac{1}{\beta_{n}^{4}\left\lfloor T \beta_{n}^{-4}\right\rfloor} Q\left[\log W_{\beta_{n},\left\lfloor T \beta_{n}^{-4}\right\rfloor}(\eta)\right] \\
& =\lim _{T \rightarrow \infty} \lim _{n \rightarrow \infty} \frac{1}{\beta_{n}^{4}\left\lfloor T \beta_{n}^{-4}\right\rfloor} Q\left[\log W_{\beta_{n},\left\lfloor T \beta_{n}^{-4}\right\rfloor}(\eta)\right] \\
& =\lim _{T \rightarrow \infty} \frac{1}{T} P_{\mathcal{Z}}\left[\log \mathcal{Z}_{\sqrt{2}}(T)\right] .
\end{aligned}
$$

Thus, the heart of our main result is just the exchangeability of limits in $n, T$ and integral.

Remark 1.3. (i) A dimension free concentration inequality assumption is used to prove the uniform integrability of $\left\{\log W_{\beta_{n},\left\lfloor T \beta_{n}^{-4}\right\rfloor}(\eta): n \in \mathbb{N}\right\}$.

(ii) The limit value $-\frac{1}{6}$ in (1.8) appears as the limit of the free energy of the continuum directed polymers (see Lemma 2.2):

$$
F_{\mathcal{Z}}(\beta)=\lim _{T \rightarrow \infty} \frac{1}{T} P_{\mathcal{Z}}\left[\log \int_{\mathbb{R}} \mathcal{Z}_{\beta}^{x}(T, y) d y\right]
$$

at $\beta=\sqrt{2}$.

$-\frac{1}{6}$ is different from the value $-\frac{1}{24}$ in the conjecture (1.6). However, since $F(\beta)$ has the scaling relation (see also Lemma 2.2), we have

$$
-\frac{1}{6}=F_{\mathcal{Z}}(\sqrt{2})=-(\sqrt{2})^{4} F_{\mathcal{Z}}(1)=-\frac{\sqrt{2}^{4}}{24} .
$$

The factor $\sqrt{2}$ which comes from the periodicity of simple random walk is missed in the conjecture (1.6) .

Remark 1.4. (i) The idea of a dimension free concentration inequality (1.7) is inspired by [14]. In [14], they obtain the sharp asymptotics of the critical curve for discrete random pinning model by comparing the partition functions of the continuum random pinning model under a similar assumption, that is they prove such an exchange of the limits $\beta \rightarrow 0$ and $N \rightarrow \infty$. Actually, the techniques for random pinning model are often applied to directed polymers in random environment. For example, the coarse-graining argument and the fractional moment method used in this paper are developed in the literature in random pinning model [41, 24] and then applied to directed polymers in random environment [28].

(ii) A dimension free concentration inequality (1.7) is fairly general. It is known that the following distribution satisfies (1.7).

(a) If $\eta(n, x)$ is bounded, then (1.7) holds with $g(t)=e^{-t^{2}}$ [30, Corollary 4.10].

(b) If the law of $\eta(n, x)$ satisfies a log-Sobolev inequality (for example Gaussian distribution), then (1.7) holds with $g(t)=e^{-t^{2}}$ [30, Theorem 5.3, Corollary 5.7]

(c) If the law of $\eta(n, x)$ has the probability density with $c_{\gamma} \exp \left(-|x|^{\gamma}\right)$, then (1.7) holds with $g(t)=e^{-|t|^{\gamma}}(\gamma \in[1,2])$ [30, Proposition 4.18, Proposition 4.19].

\subsection{Organization of this paper}

This paper is structured as follows:

- We first give the strategy of the proof of our main result in section 2 . 
- Section 3 is devoted to prove the statements mentioned in section 2 related to discrete directed polymers.

- Section 4 is also devoted to prove the statement mentioned in section 2 related to continuum directed polymers.

- Section 5 is devoted to prove the propositions which are discussed in section 4 .

- Appendix is devoted to some formulas on Gaussian heat kernels.

\section{Proof of Theorem 1.1}

In this section, we give the strategy of the proof of Theorem 1.1.

\subsection{Proof of limit inferior}

The proof of the limit inferior is simple.

From the definition of free energy (1.3), we have for any $N \geq 1$

$$
F(\beta)=\sup _{N \geq 1} \frac{1}{N} Q\left[\log W_{\beta, N}(\eta)\right] \geq \frac{1}{N} Q\left[\log W_{\beta, N}(\eta)\right] .
$$

By taking $\beta=\beta_{n}, N=\left\lfloor T \beta_{n}^{-4}\right\rfloor$ for $n \geq 1$ and $T>0$, we have that

$$
\frac{1}{\left\lfloor T \beta_{n}^{-4}\right\rfloor} Q\left[\log W_{\beta_{n},\left\lfloor T \beta_{n}^{-4}\right\rfloor}(\eta)\right] \leq F\left(\beta_{n}\right)
$$

i.e.

$$
\frac{\beta_{n}^{-4}}{\left\lfloor T \beta_{n}^{-4}\right\rfloor} Q\left[\log W_{\beta_{n},\left\lfloor T \beta_{n}^{-4}\right\rfloor}(\eta)\right] \leq \frac{1}{\beta_{n}^{4}} F\left(\beta_{n}\right) .
$$

Thus, if $\left\{\log W_{\beta_{n},\left\lfloor T \beta_{n}^{-4}\right\rfloor}(\eta): n \in \mathbb{N}\right\}$ is uniformly integrable, then we have from Theorem 1.2 that

$$
\frac{1}{T} P_{\mathcal{Z}}\left[\log \mathcal{Z}_{\sqrt{2}}(T)\right] \leq \varliminf_{n \rightarrow \infty} \frac{1}{\beta_{n}^{4}} F\left(\beta_{n}\right) .
$$

Taking the limit in $T$, we have that

$$
\lim _{T \rightarrow \infty} \frac{1}{T} P_{\mathcal{Z}}\left[\log \mathcal{Z}_{\sqrt{2}}(T)\right] \leq \varliminf_{n \rightarrow \infty} \frac{1}{\beta_{n}^{4}} F\left(\beta_{n}\right) .
$$

Therefore, it is enough to show the following lemmas.

Lemma 2.1. Suppose $d=1$. We assume (1.7). Then, for each $T>0$

$$
\left\{\log W_{\beta_{n},\left\lfloor T \beta_{n}^{-4}\right\rfloor}(\eta): n \in \mathbb{N}\right\} \text { is uniformly integrable. }
$$

Lemma 2.2. For $\beta \in \mathbb{R}$, we have the limit

$$
F_{\mathcal{Z}}(\beta)=\lim _{T \rightarrow \infty} \frac{1}{T} P_{\mathcal{Z}}\left[\log \mathcal{Z}_{\beta}(T)\right]=\sup _{T>0} \frac{1}{T} P_{\mathcal{Z}}\left[\log \mathcal{Z}_{\beta}(T)\right] .
$$

In particular, we have

$$
F_{\mathcal{Z}}(\beta)=\beta^{4} F_{\mathcal{Z}}(1)=-\frac{\beta^{4}}{24} .
$$

We should take $n=\left\lfloor\beta_{n}^{-4}\right\rfloor$ in general. However, we may consider alternatively

$$
\beta_{n}=n^{-\frac{1}{4}}
$$

case without loss of generality. Throughout the rest of paper, we take $\beta_{n}=n^{-\frac{1}{4}}$. 


\subsection{Proof of limit superior}

The proof of the limit superior is more complicated than the one of the limit inferior.

We will give an idea of the proof. The basic technique is the coarse-graining argument and the fractional moment method from [28].

It is clear by Jensen's inequality that for each $\theta \in(0,1), T \in \mathbb{N}$, and $N \in \mathbb{N}$,

$$
\begin{aligned}
\frac{1}{N T} Q\left[\log W_{\beta_{n}, N T n}(\eta)\right] & =\frac{1}{\theta N T} Q\left[\log W_{\beta_{n}, N T n}(\eta)^{\theta}\right] \\
& \leq \frac{1}{\theta N T} \log Q\left[W_{\beta_{n}, N T n}(\eta)^{\theta}\right]
\end{aligned}
$$

We will take the limit superior of both sides in $N \rightarrow \infty, n \rightarrow \infty, T \rightarrow \infty$, and then $\theta \rightarrow 0$ in this order. Then, it is clear that

$$
\varlimsup_{n \rightarrow \infty} \frac{1}{\beta_{n}^{4}} F\left(\beta_{n}\right) \leq \varlimsup_{\theta \rightarrow 0} \varlimsup_{T \rightarrow \infty} \varlimsup_{n \rightarrow \infty} \varlimsup_{N \rightarrow \infty} \frac{1}{\theta N T} \log Q\left[W_{\beta_{n}, N T n}(\eta)^{\theta}\right] .
$$

We divide $\mathbb{Z}$ into the blocks with size of order $n^{1 / 2}$ : For $y \in \mathbb{Z}$, we set

$$
B_{y}^{n}=\left[(2 y-1)\left\lfloor n^{1 / 2}\right\rfloor+y,(2 y+1)\left\lfloor n^{1 / 2}\right\rfloor+y\right] .
$$

We remark that $\left\{B_{y}^{n}: y \in \mathbb{Z}\right\}$ are disjoint and cover $\mathbb{Z}$, that is

$$
B_{x}^{n} \cap B_{y}^{n}=\emptyset \text { if } x \neq y \text { and } \bigcup_{y \in \mathbb{Z}} B_{y}^{n}=\mathbb{Z} .
$$

For each $\ell \in \mathbb{N}$, we denote by $B_{y}^{n}(\ell)$ the set of lattice points $z \in B_{y}^{n}$ such that

$$
z-\ell \in 2 \mathbb{Z}
$$

that is the set of lattices in $B_{y}^{n}$ which can be reached by random walk $\left(S, P_{S}\right)$ at time $\ell$.

We would like to estimate the right hand side in (2.4).

For $\theta \in(0,1)$, we have that

$$
Q\left[W_{\beta_{n}, N T n}(\eta)^{\theta}\right] \leq \sum_{Z} Q\left[\hat{W}_{\beta_{n}, N T n}(\eta, Z)^{\theta}\right]
$$

where for $Z=\left(z_{1}, \cdots, z_{N}\right) \in \mathbb{Z}^{N}$,

$$
\begin{aligned}
& \hat{W}_{\beta_{n}, N T n}(\eta, Z) \\
& =P_{S}\left[\prod_{i=1}^{N T n} \zeta_{i, S_{i}}\left(\beta_{n}, \eta\right): S_{\ell T n} \in B_{z_{\ell}}^{n}(\ell T n) \text { for all } \ell=1, \cdots, N\right]
\end{aligned}
$$

is the decomposition of partition function by the sequence of blocks where $S$ passes through for time $k=\operatorname{Tni}(i=1, \cdots, N)$ and we have used the fact $(a+b)^{\theta} \leq a^{\theta}+b^{\theta}$ for $a, b \geq 0$ and $\theta \in(0,1)$. Then, we have from the Markov property and shift invariance of the environment that

$$
Q\left[W_{\beta_{n}, N T n}^{\theta}(\eta)\right] \leq\left(\sum_{z \in \mathbb{Z}} Q\left[\max _{x \in B_{0}^{n}(0)} P_{S}^{x}\left[\prod_{i=1}^{T n} \zeta_{i, S_{i}}\left(\beta_{n}, \eta\right): S_{T n} \in B_{z}^{n}\right]\right)^{\theta}\right.
$$


Taking limit in $N$ in (2.4) with (2.5), we have that

$$
\frac{1}{\beta_{n}^{4}} F\left(\beta_{n}\right) \leq \frac{1}{\theta T} \log \sum_{z \in \mathbb{Z}} Q\left[\max _{x \in B_{0}^{n}(0)} P_{S}^{x}\left[\prod_{i=1}^{T n} \zeta_{i, S_{i}}\left(\beta_{n}, \eta\right): S_{T n} \in B_{z}^{n}\right]^{\theta}\right] .
$$

We will divide the summation in the right hand side into two parts $|z| \geq T^{2}$ and $|z|<T^{2}$.

For $|z|<T^{2}$, we bound each term by

$$
Q\left[\max _{x \in B_{0}^{n}(0)} P_{S}^{x}\left[\prod_{i=1}^{T n} \zeta_{i, S_{i}}\left(\beta_{n}, \eta\right)\right]^{\theta}\right]
$$

which we may believe from Theorem 1.2 that it converges to $P_{\mathcal{Z}}\left[\sup _{x \in[-1,1]}\left(\mathcal{Z}_{\sqrt{2}}^{x}(T)\right)^{\theta}\right]$. Indeed, we have the following lemma.

Lemma 2.3. We have that

$$
\lim _{n \rightarrow \infty} Q\left[\max _{x \in B_{0}^{n}(0)} P_{S}^{x}\left[\prod_{i=1}^{T n} \zeta_{i, S_{i}}\left(\beta_{n}, \eta\right)\right]^{\theta}\right]=P_{\mathcal{Z}}\left[\sup _{x \in[-1,1]}\left(\mathcal{Z}_{\sqrt{2}}^{x}(T)\right)^{\theta}\right] .
$$

Also, we should estimate $\sum_{|z| \geq T^{2}} Q\left[\max _{x \in B_{0}^{n}(0)} P_{S}^{x}\left[\prod_{i=1}^{T n} \zeta_{i, S_{i}}\left(\beta_{n}, \eta\right): S_{T n} \in B_{z}^{n}\right]^{\theta}\right]$. Since

$$
Q\left[P_{S}^{x}\left[\prod_{i=1}^{T n} \zeta_{i, S_{i}}\left(\beta_{n}, \eta\right): S_{T n} \in B_{z}^{n}\right]\right]=P_{S}^{x}\left(S_{T n} \in B_{z}^{n}\right)
$$

we obtain by Hölder's inequality that

$$
Q\left[P_{S}^{x}\left[\prod_{i=1}^{T n} \zeta_{i, S_{i}}\left(\beta_{n}, \eta\right): S_{T n} \in B_{z}^{n}\right]^{\theta}\right] \leq P_{S}^{x}\left(S_{T n} \in B_{z}^{n}\right)^{\theta}
$$

which decays like $\exp \left(-\frac{|z|^{2}}{T}\right)$ in $|z|$ so we may believe that

$$
\sum_{|z| \geq T^{2}} Q\left[\max _{x \in B_{0}^{n}(0)} P_{S}^{x}\left[\prod_{i=1}^{T n} \zeta_{i, S_{i}}\left(\beta_{n}, \eta\right): S_{T n} \in B_{z}^{n}\right]^{\theta}\right]
$$

decays like $\exp \left(-\frac{|T|^{4}}{T}\right)$. Rigorously, we will confirm the followings.

Lemma 2.4. There exist some constant $C_{\theta, 1}>0$ and $C_{\theta, 2}>0$ which depends on $\theta$ such that

$$
\sum_{|z| \geq T^{2}} Q\left[\max _{x \in B_{0}^{n}(0)} P_{S}^{x}\left[\prod_{i=1}^{T n} \zeta_{i, S_{i}}\left(\beta_{n}, \eta\right): S_{T n} \in B_{z}^{n}\right]^{\theta}\right] \leq C_{\theta, 1} \exp \left(-C_{\theta, 2} T^{3}\right)
$$

for any $n \geq 1$.

Thus, we have that

$$
\varlimsup_{n \rightarrow \infty} \frac{1}{\beta_{n}^{4}} F\left(\beta_{n}\right) \leq \frac{1}{\theta T} \log \left(C_{\theta, 1} \exp \left(-C_{\theta, 2} T^{3}\right)+2 T^{2} P_{\mathcal{Z}}\left[\sup _{x \in[-1,1]}\left(\mathcal{Z}_{\sqrt{2}}^{x}(T)\right)^{\theta}\right]\right) .
$$

The following result gives us an upper bound of the limit superior: 
Lemma 2.5. We have that

$$
\varlimsup_{\theta \rightarrow 0} \varlimsup_{T \rightarrow \infty} \frac{1}{T \theta} \log P_{\mathcal{Z}}\left[\sup _{x \in[-1,1]}\left(\mathcal{Z}_{\sqrt{2}}^{x}(T)\right)^{\theta}\right] \leq F_{\mathcal{Z}}(\sqrt{2}) .
$$

In the rest of the paper, we will prove the above lemmas.

\section{Proof of Lemma 2.1, Lemma 2.3, and Lemma 2.4}

I this subsection, we will give the proofs of lemmas related to directed polymers in random environment.

\subsection{Proof of Lemma 2.1}

We take $\mathbb{R}^{m}$ as $\mathbb{R}^{E_{n}}$ in the proof of Lemma 2.1 with $E_{n}=\{1, \cdots, T n\} \times\{-T n, \cdots, T n\}$ which contains all time-space points where simple random walk can reach up to time $T n$.

Proof of Lemma 2.1. Since $W_{\beta_{n}, T n}(\eta)$ is $L^{2}$-bounded from Theorem $1.2,\left\{\log ^{+} W_{\beta_{n}, T n}(\eta)\right.$ : $n \in \mathbb{N}\}$ is uniformly integrable so that it is enough to see that $\left\{\log ^{-} W_{\beta_{n}, T n}(\eta): n \in \mathbb{N}\right\}$ is uniformly integrable, where we set

$$
\log ^{+} x=\left\{\begin{array}{ll}
\log x, & x \in[1, \infty) \\
0, & x \in(0,1)
\end{array}, \quad \log ^{-} x=\left\{\begin{array}{ll}
0, & x \in[1, \infty) \\
-\log x, & x \in(0,1)
\end{array} .\right.\right.
$$

The uniform integrability of $\left\{\log ^{-} W_{\beta_{n}, T n}(\eta): n \in \mathbb{N}\right\}$ is equivalent to

$$
\lim _{M \rightarrow \infty} \sup _{n \in \mathbb{N}} \int_{M}^{\infty} Q\left(-\log W_{\beta_{n}, T n}(\eta)>t\right) d t=0 .
$$

We will prove (3.1) under the assumption (1.7).

When we look at $W_{\beta_{n}, T n}(\eta)$ as the function of $\left\{\eta(i, x):(i, x) \in E_{n}\right\}, \log W_{\beta_{n}, T n}(\eta)$ is differentiable and convex. Indeed, we have that

$$
\frac{\partial}{\partial \eta(i, x)} \log W_{\beta_{n}, T n}(\eta)=\frac{1}{W_{\beta_{n}, T n}(\eta)} P_{S}\left[\beta_{n} \prod_{k=1}^{T n} \zeta_{k, S_{k}}\left(\beta_{n}, \eta\right): S_{i}=x\right]
$$

and for $s \in[0,1]$, for $\eta=\left\{\eta(i, x):(i, x) \in E_{n}\right\}$ and $\eta^{\prime}=\left\{\eta^{\prime}(i, x):(i, x) \in E_{n}\right\}$,

$$
\begin{aligned}
W_{\beta_{n}, T n}\left(s \eta+(1-s) \eta^{\prime}\right) & =P_{S}\left[\prod_{k=1}^{T n} \zeta_{k, S_{k}}\left(\beta_{n}, s \eta+(1-s) \eta^{\prime}\right)\right] \\
& \leq W_{\beta_{n}, T n}(\eta)^{s} W_{\beta_{n}, T n}\left(\eta^{\prime}\right)^{1-s}
\end{aligned}
$$

Thus, we can take $f(\eta)=\log W_{\beta_{n}, T n}(\eta)$ in (1.7). Then, we have that

$$
\begin{aligned}
& Q\left(\log W_{\beta_{n}, T n}(\eta)<a-t\right) \\
& \leq Q\left(\log W_{\beta_{n}, T n}(\eta)>a,\left|\nabla \log W_{\beta_{n}, T n}(\eta)\right| \leq c\right)^{-1} g\left(\frac{t}{c}\right), \quad a \in \mathbb{R}, t, c \in(0, \infty) .
\end{aligned}
$$

We will set $a=-\log 2$ and Proposition 3.1 below yields (3.1). Indeed, we have from Proposition 3.1 and (3.2) that there exists $C>0$ such that

$$
\int_{M}^{\infty} Q\left(-\log W_{\beta_{n}, T n}(\eta)>t\right) d t \leq C \int_{M+\log 2}^{\infty} g\left(\frac{t}{c}\right) d t
$$

which uniformly converges to 0 as $M \rightarrow \infty$. 
Proposition 3.1. Suppose (1.7). Then, there exists some $c>0$ such that

$$
\left\{Q\left(W_{\beta_{n}, T n}(\eta)>\frac{1}{2},\left|\nabla \log W_{\beta_{n}, T n}(\eta)\right| \leq c\right)^{-1}: n \in \mathbb{N}\right\} \text { is bounded. }
$$

The idea of the proof comes from [15] and [32].

Proof. It is easy to see that

$$
\begin{aligned}
\left|\nabla \log W_{\beta_{n}, T n}(\eta)\right|^{2} & =\beta_{n}^{2} \sum_{(i, x) \in E_{n}}\left(\frac{1}{W_{\beta_{n}, T n}(\eta)} P_{S}\left[\prod_{k=1}^{T n} \zeta_{k, S_{k}}\left(\beta_{n}, \eta\right): S_{i}=x\right]\right)^{2} \\
& =\beta_{n}^{2} \sum_{(i, x) \in E_{n}} \mu_{\beta_{n}, T n}^{\eta}\left(S_{i}=x\right)^{2} \\
& =\beta_{n}^{2}\left(\mu_{\beta_{n}, T n}^{\eta}\right)^{\otimes 2}\left[\sharp\left\{1 \leq i \leq T n: S_{i}=S_{i}^{\prime}\right\}\right],
\end{aligned}
$$

where $\mu_{\beta, n}^{\eta}$ is the probability measure on the simple random walk paths defined by

$$
\mu_{\beta, n}^{\eta}(s)=\frac{1}{W_{\beta, n}(\eta)} \exp \left(\beta \sum_{i=1}^{n} \eta\left(i, s_{i}\right)-n \lambda(\beta)\right) P_{S}\left(S_{[0, n]}=s\right)
$$

for $s=\left(s_{0}, s_{1}, \cdots, s_{n}\right) \in \mathbb{Z}^{n+1},\left(\mu_{\beta_{n}, T n}^{\eta}\right)^{\otimes 2}$ is the product probability measure of $\mu_{\beta_{n}, T n^{\prime}}^{\eta}$ and $S$ and $S^{\prime}$ are two independent directed polymers each of whose law is $\mu_{\beta_{n}, T n}^{\eta}$.

We write

$$
L_{n}\left(s, s^{\prime}\right)=\sharp\left\{1 \leq i \leq n: s_{i}=s_{i}^{\prime}\right\}
$$

for $s=\left(s_{1}, \cdots, s_{n}\right)$ and $s^{\prime}=\left(s_{1}^{\prime}, \cdots, s_{n}^{\prime}\right) \in \mathbb{Z}^{n}$.

We define the event $A_{n}$ on the environment by

$$
A_{n}=\left\{\eta: W_{\beta_{n}, T n}(\eta) \geq \frac{1}{2}, \beta_{n}^{2}\left(\mu_{\beta_{n}, T n}^{\eta}\right)^{\otimes 2}\left[L_{T n}\left(S, S^{\prime}\right)\right] \leq C_{1}\right\}
$$

for some $C_{1}>0$ which we will take large enough later. We claim that for $C_{1}>0$ large enough, there exists a constant $\delta>0$ such that

$$
Q\left(A_{n}\right)>\delta
$$

for all $n \geq 1$.

We observe that

$$
\begin{aligned}
Q\left(A_{n}\right)= & Q\left(\left\{\eta: W_{\beta_{n}, T n}(\eta) \geq \frac{1}{2}\right\}\right) \\
& -Q\left(\left\{\eta: W_{\beta_{n}, T n}(\eta) \geq \frac{1}{2}, \beta_{n}^{2}\left(\mu_{\beta_{n}, T n}^{\eta}\right)^{\otimes 2}\left[L_{\lfloor T n}\left(S, S^{\prime}\right)\right]>C_{1}\right\}\right) \\
\geq & Q\left(\left\{\eta: W_{\beta_{n}, T n}(\eta) \geq \frac{1}{2}\right\}\right) \\
& -Q\left(\left\{\eta: P_{S, S^{\prime}}\left[\beta_{n}^{2} L_{T n}\left(S, S^{\prime}\right) \prod_{i=1}^{T n} \zeta_{i, S_{i}}\left(\beta_{n}, \eta\right) \zeta_{i, S_{i}}\left(\beta_{n}, \eta\right)\right]>\frac{C_{1}}{4}\right\}\right) \\
\geq & Q\left(\left\{\eta: W_{\beta_{n}, T n}(\eta) \geq \frac{1}{2}\right\}\right) \\
& -\frac{4}{C_{1}} Q\left[P_{S, S^{\prime}}\left[\beta_{n}^{2} L_{T n}\left(S, S^{\prime}\right) \prod_{i=1}^{T n} \zeta_{i, S_{i}}\left(\beta_{n}, \eta\right) \zeta_{i, S_{i}^{\prime}}\left(\beta_{n}, \eta\right)\right]\right],
\end{aligned}
$$


where we have used the Chebyshev's inequality and $\left(S^{\prime}, P_{S^{\prime}}\right)$ is the simple random walk on $\mathbb{Z}$ starting from the origin and $P_{S, S^{\prime}}$ is the product measure of $P_{S}$ and $P_{S^{\prime}}$.

Recalling that $Q\left[W_{\beta_{n}, T n}(\eta)\right]=1$, Paley-Zygmund's inequality yields that

$$
\begin{aligned}
Q\left(\left\{\eta: W_{\beta_{n}, T n}(\eta) \geq \frac{1}{2}\right\}\right) & =Q\left(\left\{\eta: W_{\beta_{n}, T n}(\eta) \geq \frac{1}{2} Q\left[W_{\beta_{n}, T n}(\eta)\right]\right\}\right) \\
& \geq \frac{1}{4} \frac{\left(Q\left[W_{\beta_{n}, T n}(\eta)\right]\right)^{2}}{Q\left[W_{\beta_{n}, T n}(\eta)^{2}\right]} \\
& =\frac{1}{4} \frac{1}{Q\left[W_{\beta_{n}, T n}(\eta)^{2}\right]} .
\end{aligned}
$$

The $L^{2}$-boundedness of $W_{\beta_{n}, T n}(\eta)$ (see Theorem 1.2) implies that there exists $C_{2}>0$ such that for all $n \geq 1$

$$
Q\left[W_{\beta_{n}, T n}(\eta)^{2}\right] \leq C_{2} .
$$

Hence, we have that for all $n \geq 1$

$$
Q\left(\left\{\eta: W_{\beta_{n}, T n}(\eta) \geq \frac{1}{2}\right\}\right) \geq \frac{1}{4 C_{2}} .
$$

By Fubini's theorem, we have that

$$
\begin{aligned}
& Q\left[P_{S, S^{\prime}}\left[\beta_{n}^{2} L_{T n}\left(S, S^{\prime}\right) \prod_{i=1}^{T n} \zeta_{i, S_{i}}\left(\beta_{n}, \eta\right) \zeta_{i, S_{i}^{\prime}}\left(\beta_{n}, \eta\right)\right]\right] \\
& =P_{S, S^{\prime}}\left[\beta_{n}^{2} L_{T n}\left(S, S^{\prime}\right) \exp \left(\left(\lambda\left(2 \beta_{n}\right)-2 \lambda\left(\beta_{n}\right)\right) L_{T n}\left(S, S^{\prime}\right)\right)\right]
\end{aligned}
$$

and

$$
\begin{aligned}
& Q\left[W_{\beta_{n}, T n}(\eta)^{2}\right] \\
& =Q\left[P_{S, S^{\prime}}\left[\prod_{i=1}^{T n} \zeta_{i, S_{i}}\left(\beta_{n}, \eta\right) \zeta_{i, S_{i}^{\prime}}\left(\beta_{n}, \eta\right)\right]\right] \\
& =P_{S, S^{\prime}}\left[\exp \left(\left(\lambda\left(2 \beta_{n}\right)-2 \lambda\left(\beta_{n}\right)\right) L_{T n}\left(S, S^{\prime}\right)\right)\right] .
\end{aligned}
$$

We will see that there exists a constant $r>0$ such that

$$
P_{S, S^{\prime}}\left[\beta_{n}^{2} L_{T n}\left(S, S^{\prime}\right) \exp \left(\left(\lambda\left(2 \beta_{n}\right)-2 \lambda\left(\beta_{n}\right)\right) L_{T n}\left(S, S^{\prime}\right)\right)\right] \leq Q\left[W_{r \beta_{n}, T n}(\eta)^{2}\right]
$$

for any $n$ large enough. If (3.6) holds, then the $L^{2}$-boundedness of $W_{\beta_{n}, T n}(\eta)$ also implies that there exists $C_{3}>0$ such that

$$
Q\left[P_{S, S^{\prime}}\left[\beta_{n}^{2} L_{T n}\left(S, S^{\prime}\right) \prod_{i=1}^{T n} \zeta_{i, S_{i}}\left(\beta_{n}, \eta\right) \zeta_{i, S_{i}^{\prime}}\left(\beta_{n}, \eta\right)\right]\right] \leq C_{3}
$$

and combining this with (3.4) and (3.5), we have that

$$
Q\left(\eta \in A_{n}\right) \geq \frac{1}{4 C_{2}}-\frac{4 C_{3}}{C_{1}}
$$

and we obtain (3.3) by taking $C_{3}>0$ large enough.

Since

$$
\frac{\lambda\left(2 \beta_{n}\right)-2 \lambda\left(\beta_{n}\right)}{\beta_{n}^{2}} \rightarrow \lambda^{\prime \prime}(0)=1
$$


we have that

$$
\beta_{n}^{2} \leq 2\left(\lambda\left(2 \beta_{n}\right)-2 \lambda\left(\beta_{n}\right)\right)
$$

for $n$ large enough and hence it holds by the fact $e^{x} \geq x$ for $x \in \mathbb{R}$ that

$$
\begin{aligned}
& P_{S, S^{\prime}}\left[\beta_{n}^{2} L_{T n}\left(S, S^{\prime}\right) \exp \left(\left(\lambda\left(2 \beta_{n}\right)-2 \lambda\left(\beta_{n}\right)\right) L_{T n}\left(S, S^{\prime}\right)\right)\right] \\
& \leq P_{S, S^{\prime}}\left[\exp \left(3\left(\lambda\left(2 \beta_{n}\right)-2 \lambda\left(\beta_{n}\right)\right) L_{T n}\left(S, S^{\prime}\right)\right)\right]
\end{aligned}
$$

for any $n$ large enough. Also, we have

$$
\frac{\lambda\left(2 r \beta_{n}\right)-2 \lambda\left(r \beta_{n}\right)}{\lambda\left(2 \beta_{n}\right)-2 \lambda\left(\beta_{n}\right)} \rightarrow r^{2}
$$

as $n \rightarrow \infty$ for $r>0$ and hence (3.6) follows.

\subsection{Proof of Lemma 2.3}

We define for $x, y \in \mathbb{Z}$

$$
W_{\beta, n}^{x}(\eta)=P_{S}^{x}\left[\prod_{k=1}^{n} \zeta_{k, S_{k}}(\beta, \eta)\right], \quad W_{\beta, n}^{x}(\eta, y)=P_{S}^{x}\left[\prod_{k=1}^{n} \zeta_{k, S_{k}}(\beta, \eta): S_{n}=y\right]
$$

and for $u \in[-1,1]$

$$
f_{n}(u):=W_{\beta_{n}, T n}^{u n^{1 / 2}}(\eta)= \begin{cases}W_{\beta_{n}, T n}^{u n^{1 / 2}}(\eta), & u n^{1 / 2} \in B_{0}^{n}(0) \\ \text { linear interpolation, } & \text { otherwise }\end{cases}
$$

Since the finite dimensional distributions $\left\{W_{\beta_{n}, T n}^{x_{i} n^{1 / 2}}(\eta): 1 \leq i \leq m\right\}$ for $x_{1} n^{1 / 2}, \cdots, x_{m} n^{1 / 2} \in B_{0}^{n}(0)$ converge to $\left\{\mathcal{Z}_{\sqrt{2}}^{x_{i}}(T): 1 \leq i \leq m\right\}$ (see [2, Section 6.2]), the tightness of $\left\{f_{n}(x)^{\theta}: x \in[-1,1]\right\}$ in $C[-1,1]$ and $L^{p}$-boundedness of $\max _{x \in B_{0}^{n}(0)} W_{\beta_{n}, T n}^{x}(\eta)^{\theta}$ for some $p>1$ imply Lemma 2.3.

We will use Garsia-Rodemich-Rumsey's lemma [36, Lemma A.3.1] many times throughout this paper.

Lemma 3.2. Let $\phi:[0, \infty) \rightarrow[0, \infty)$ and $\Psi:[0, \infty) \rightarrow[0, \infty)$ be continuous and strictly increasing functions satisfying

$$
\phi(0)=\Psi(0)=0, \lim _{t \rightarrow \infty} \Psi(t)=\infty
$$

Let $f: \mathbb{R}^{d} \rightarrow \mathbb{R}$ be a continuous function. Provided

$$
\Gamma=\int_{B_{r}(x)} \int_{B_{r}(x)} \Psi\left(\frac{|f(t)-f(s)|}{\phi(|t-s|)}\right) d s d t<\infty
$$

where $B_{r}(x)$ is an open ball in $\mathbb{R}^{d}$ centered at $x$ with radius $r$, then for all $s, t \in B_{r}(x)$,

$$
|f(t)-f(s)| \leq 8 \int_{0}^{2|t-s|} \Psi^{-1}\left(\frac{4^{d+1} \Gamma}{\lambda_{d} u^{2 d}}\right) \phi(d u)
$$

where $\lambda_{d}$ is a universal constant depending only on $d$. 
Applying Lemma 3.2 with $\Psi(x)=|x|^{p}, \phi(u)=u^{q}$ for $p \geq 1, q>0$ and $p q>2 d$, we have that

$$
|f(t)-f(s)| \leq \frac{2^{\frac{2}{p}+q+3} q}{\lambda_{d}^{\frac{1}{p}}\left(q-\frac{2 d}{p}\right)}|t-s|^{q-\frac{2 d}{p}}\left(\int_{B_{r}(x)} \int_{B_{r}(x)}\left(\frac{|f(v)-f(w)|}{|v-w|^{q}}\right)^{p} d v d w\right)^{\frac{1}{p}}
$$

for $t, s \in B_{r}(x)(r>0)$. In particular, if $t, s \in B_{r}(x)$ satisfies $|t-s|<\delta$ for some $\delta>0$, then

$$
|f(t)-f(s)| \leq C_{p, q, d}|\delta|^{q-\frac{2 d}{p}}\left(\int_{B_{r}(x)} \int_{B_{r}(x)}\left(\frac{|f(v)-f(w)|}{|v-w|^{q}}\right)^{p} d v d w\right)^{\frac{1}{p}}
$$

and hence

$$
\sup _{t \in B_{r}(x)}|f(t)| \leq|f(x)|+C_{p, q, d} r^{q-\frac{2 d}{p}}\left(\int_{B_{r}(x)} \int_{B_{r}(x)}\left(\frac{|f(v)-f(w)|}{|v-w|^{q}}\right)^{p} d v d w\right)^{\frac{1}{p}} .
$$

Proof of Lemma 2.3. It is enough to show the tightness of $\left\{f_{n}(x)^{\theta}: x \in[-1,1]\right\}$ in $C[-1,1]$ and $L^{2 / \theta}$-boundedness of $\max _{x \in[-1,1]} W_{\beta_{n}, T n}^{x n^{1 / 2}}(\eta)^{\theta}$.

We apply (3.7) to $f_{n}(u)^{\theta}$. Then, it follows that

$$
\max _{x \in B_{0}^{n}(0)}\left(W_{\beta_{n}, T n}^{x}(\eta)\right)^{\theta} \leq\left(W_{\beta_{n}, T n}(\eta)\right)^{\theta}+C_{p, q} B_{p, q, n, \theta}
$$

where

$$
B_{p, q, n, \theta}=\left(\int_{-1}^{1} \int_{-1}^{1}\left(\frac{\left|f_{n}(v)^{\theta}-f_{n}(w)^{\theta}\right|}{|v-w|^{q}}\right)^{p} d v d w\right)^{\frac{1}{p}}
$$

We have the following proposition whose proof will be given in the end of this subsection.

Proposition 3.3. For $p=\frac{5}{\theta}>1$, there exist $C_{T, \theta}>0$ and $\eta_{p, \theta}=\frac{p \theta}{2}=\frac{5}{2}$ such that

$$
Q\left[\left|f_{n}(v)^{\theta}-f_{n}(w)^{\theta}\right|^{p}\right] \leq C_{T, \theta}|v-w|^{\eta_{p, \theta}},
$$

for $v, w \in[-1,1]$.

To prove Lemma 2.3, we set $p=\frac{5}{\theta}>1, q=\frac{2 \theta}{3}>0$ with $p q=\frac{10}{3}>2$ and $\eta_{p, \theta}=\frac{p \theta}{2}=\frac{5}{2}$ which satisfies $\eta_{p, \theta}-p q=-\frac{5}{6}>-1$ in the following.

Tightness of $\left\{f_{n}(x)^{\theta}: x \in[-1,1]\right\}$ in $C[-1,1]$ : It follows from (3.8) and (3.11) that for any $\delta>0$

$$
\begin{aligned}
& Q\left[\sup _{\substack{t, s \in[-1,1] \\
|t-s|<\delta}}\left|f_{n}(t)^{\theta}-f_{n}(s)^{\theta}\right|\right] \\
& \leq C_{p, q, 1}|\delta|^{q-\frac{2}{p}}\left(\int_{-1}^{1} \int_{-1}^{1} Q\left[\left(\frac{\left|f_{n}(v)^{\theta}-f_{n}(w)^{\theta}\right|}{|v-w|^{q}}\right)^{p}\right] d v d w\right)^{\frac{1}{p}} \\
& \leq C_{T, \theta, 1}|\delta|^{q-\frac{2}{p}}
\end{aligned}
$$


and hence, we have from the $L^{2}$-boundedness of $f_{n}(0)$ that for any $\varepsilon>0$

$$
\varlimsup_{\delta \rightarrow 0} \sup _{n \geq 1} Q\left(\sup _{\substack{t, s \in[-1,1] \\|t-s|<\delta}}\left|f_{n}(t)^{\theta}-f_{n}(s)^{\theta}\right|>\varepsilon\right)=0
$$

and

$$
\varlimsup_{\lambda \rightarrow \infty} \sup _{n \geq 1} Q\left(f_{n}(0)^{\theta}>\lambda\right)=0
$$

so that the tightness of $\left\{f_{n}(x)^{\theta}: x \in[-1,1]\right\}$ in $C[-1,1]$ holds (see [27, Chapter 2, Theorem 4.10]).

$L^{2 / \theta}$-boundedness of $\max _{x \in[-1,1]} W_{\beta_{n}, T n}^{x n^{1 / 2}}(\eta)^{\theta}:$ It follows from (3.10) that

$$
\begin{aligned}
& Q\left[\left(\max _{x \in B_{0}^{n}(0)}\left(W_{\beta_{n}, T n}^{x}(\eta)\right)^{\theta}\right)^{2 / \theta}\right]^{\theta / 2} \\
& \leq Q\left[\left(\left(W_{\beta_{n}, T n}(\eta)\right)^{\theta}\right)^{2 / \theta}\right]^{\theta / 2}+C_{p, q} Q\left[B_{p, q, n, \theta}^{2 / \theta}\right]^{\theta / 2} .
\end{aligned}
$$

The first term in the right hand side is bounded from Theorem 1.2. Also, we have from Proposition 3.3 that

$$
\begin{aligned}
Q\left[B_{p, q, n, \theta}^{2 / \theta}\right]^{\theta / 2} & =Q\left[\left(\int_{-1}^{1} \int_{-1}^{1}\left(\frac{\left|f_{n}(v)^{\theta}-f_{n}(w)^{\theta}\right|}{|v-w|^{q}}\right)^{p} d v d w\right)^{\frac{\theta}{5} \frac{2}{\theta}}\right]^{\frac{\theta}{2}} \\
& \leq Q\left[\left(\int_{-1}^{1} \int_{-1}^{1}\left(\frac{\left|f_{n}(v)^{\theta}-f_{n}(w)^{\theta}\right|}{|v-w|^{q}}\right)^{p} d v d w\right)\right]^{\frac{\theta}{5}} \\
& \leq C_{T, \theta, 2} .
\end{aligned}
$$

Thus, the $L^{2 / \theta}$-boundedness of $\max _{x \in[-1,1]} W_{\beta_{n}, T n}^{x n^{1 / 2}}(\eta)^{\theta}$ follows and therefore Lemma 2.3 follows.

Proof of Proposition 3.3. We remark that

$$
\left|f_{n}(t)^{\theta}-f_{n}(s)^{\theta}\right| \leq\left|W_{\beta_{n}, T n}^{t n^{1 / 2}}(\eta)-W_{\beta_{n}, T n}^{s n^{1 / 2}}(\eta)\right|^{\theta},
$$

where we have used that $(x+y)^{\theta} \leq x^{\theta}+y^{\theta}$ for $x \geq 0, y \geq 0$ so that for $p \geq 1$,

$$
Q\left[\left|f_{n}(t)^{\theta}-f_{n}(s)^{\theta}\right|^{p}\right] \leq Q\left[\left|W_{\beta_{n}, T n}^{t n^{1 / 2}}(\eta)-W_{\beta_{n}, T n}^{s n^{1 / 2}}(\eta)\right|^{p \theta}\right] .
$$

First, we will estimate

$$
Q\left[\left|W_{\beta_{n}, T n}^{x}(\eta)-W_{\beta_{n}, T n}^{y}(\eta)\right|^{2}\right],
$$

for $x, y \in B_{0}^{n}(0)$. When we define i.i.d. random variables by

$$
e_{n}(k, x)=\exp \left(\beta_{n} \eta(k, x)-\lambda\left(\beta_{n}\right)\right)-1, \quad(k, x) \in \mathbb{N} \times \mathbb{Z},
$$

we find that

$$
Q\left[e_{n}(k, x)\right]=0, \text { and } \frac{Q\left[e_{n}(k, x)^{2}\right]}{\beta_{n}^{2}}=\frac{e\left(\lambda\left(2 \beta_{n}\right)-2 \lambda\left(\beta_{n}\right)\right)-1}{\beta_{n}^{2}} \rightarrow 1 .
$$


Then, we can write

$$
\begin{aligned}
W_{\beta_{n}, T n}^{x}(\eta) & =P_{S}^{x}\left[\prod_{i=1}^{T n}\left(1+e_{n}\left(i, S_{i}\right)\right)\right] \\
& =1+\sum_{k=1}^{T n} \sum_{1 \leq i_{1}<\cdots<i_{k} \leq T n} \sum_{\mathbf{x} \in \mathbb{Z}^{k}} \prod_{j=1}^{k} p_{i_{j}-i_{j-1}}\left(x_{j}-x_{j-1}\right) e_{n}\left(i_{j}, x_{j}\right) \\
& =\sum_{k=0}^{T n} \Theta^{(k)}(x),
\end{aligned}
$$

where $p_{n}(y)=P_{S}\left(S_{n}=y\right)$ for $(n, y) \in \mathbb{N} \times \mathbb{Z}, x_{0}=x, \mathbf{x}=\left(x_{1}, \cdots, x_{k}\right)$, and

$$
\Theta^{(k)}(x)= \begin{cases}1, & k=0 \\ \sum_{1 \leq i_{1}<\cdots<i_{k} \leq T n} \sum_{\mathbf{x} \in \mathbb{Z}^{k}} \prod_{j=1}^{k} p_{i_{j}-i_{j-1}}\left(x_{j}-x_{j-1}\right) e_{n}\left(i_{j}, x_{j}\right), & k \geq 1 .\end{cases}
$$

Then, it is easy to see that

$$
Q\left[\Theta^{(k)}(x)\right]=0, \quad k \geq 1
$$

and

$$
Q\left[\Theta^{(k)}(x) \Theta^{(\ell)}(y)\right]=0, \quad k \neq \ell, \quad x, y \in \mathbb{Z} .
$$

Thus, we have that

$$
\begin{aligned}
& Q\left[\left|W_{\beta_{n}, T n}^{x}(\eta)-W_{\beta_{n}, T n}^{y}(\eta)\right|^{2}\right] \\
& =\sum_{k=1}^{T n} Q\left[\left(\Theta^{(k)}(x)-\Theta^{(k)}(y)\right)^{2}\right] \\
& =\sum_{k=1}^{T n}\left(Q\left[e_{n}(0,0)^{2}\right]\right)^{k} \sum_{1 \leq i_{1}<\cdots<i_{k} \leq T n} \sum_{\mathbf{x} \in \mathbb{Z}^{k}}\left(p_{i_{1}}\left(x, x_{1}\right)-p_{i_{1}}\left(y, x_{1}\right)\right)^{2} \prod_{j=2}^{k} p_{i_{j}-i_{j-1}}\left(x_{j-1}, x_{j}\right)^{2},
\end{aligned}
$$

where $p_{n}(x, y)=P_{S}^{x}\left(S_{n}=y\right)$ for $x, y \in \mathbb{Z}^{d}$.

Combining (3.12) with the fact that for $k \geq 1$

$$
\frac{1}{n^{k / 2}} \sum_{1 \leq i_{1}<\cdots<i_{k} \leq T n} \sum_{\mathbf{x} \in \mathbb{Z}^{k}} \prod_{j=1}^{k} p_{i_{j}-i_{j-1}}\left(x_{j}-x_{j-1}\right)^{2} \leq \frac{C_{4}^{k} T^{k / 2}}{\Gamma\left(\frac{k}{2}+1\right)},
$$

where $\Gamma(s)$ is a Gamma function at $s>0$ [2, Section 3.4 and Lemma A.1], we have

$$
\begin{aligned}
& Q\left[\left(\Theta^{(k)}(x)-\Theta^{(k)}(y)\right)^{2}\right] \\
& \leq \sum_{i=1}^{T n} \sum_{z \in \mathbb{Z}}\left(p_{i}(z-x)-p_{i}(z-y)\right)^{2} Q\left[e_{n}(0,0)^{2}\right] \frac{C_{5}^{k-1} T^{\frac{k-1}{2}}}{\Gamma\left(\frac{k-1}{2}+1\right)} \\
& =2 Q\left[e_{n}(0,0)^{2}\right] \frac{C_{5}^{k-1} T^{\frac{k-1}{2}}}{\Gamma\left(\frac{k-1}{2}+1\right)} \sum_{1 \leq i \leq T n}\left(p_{2 i}(0)-p_{2 i}(x-y)\right) .
\end{aligned}
$$


Since we know that there exists a constant $c>0$ such that for all $i \geq 1$ and $x, y \in \mathbb{Z}$ with $y \in 2 \mathbb{Z}$,

$$
\left|p_{i}(x+y)-p_{i}(x)\right| \leq \frac{c|y|}{i^{3 / 2}}+\frac{2}{\sqrt{2 \pi i}}\left|\exp \left(-\frac{(x+y)^{2}}{2 i}\right)-\exp \left(-\frac{x^{2}}{2 i}\right)\right|
$$

(see [29, Theorem 2.3.6]) and in particular for the case $x=0$,

$$
\left|p_{2 i}(0)-p_{2 i}(y)\right| \leq \frac{1}{\sqrt{\pi i}}\left|1-\exp \left(-\frac{y^{2}}{4 i}\right)\right| \leq \frac{1}{\sqrt{\pi i}}\left(\frac{y^{2}}{i} \wedge 1\right),
$$

we have that

$$
\sum_{i=1}^{\infty}\left|p_{2 i}(0)-p_{2 i}(y)\right| \leq c|y|
$$

and therefore

$$
Q\left[\left(\Theta^{(k)}(x)-\Theta^{(k)}(y)\right)^{2}\right] \leq C\left|\frac{x-y}{\sqrt{n}}\right| \frac{C_{5}^{k-1} T^{\frac{k-1}{2}}}{\Gamma\left(\frac{k-1}{2}+1\right)}
$$

and

$$
Q\left[\left|W_{\beta_{n}, T n}^{x}(\eta)-W_{\beta_{n}, T n}^{y}(\eta)\right|^{2}\right] \leq C_{T, 1}\left|\frac{x-y}{\sqrt{n}}\right|
$$

for $x, y \in B_{0}^{n}(0)$, where we remark that

$$
C_{T, 1}=C \sum_{k \geq 1} \frac{C_{5}^{k-1} T^{\frac{k-1}{2}}}{\Gamma\left(\frac{k-1}{2}+1\right)}
$$

Now, we would like to estimate

$$
Q\left[\left|W_{\beta_{n}, T n}^{t n^{1 / 2}}(\eta)-W_{\beta_{n}, T n}^{s n^{1 / 2}}(\eta)\right|^{p \theta}\right]
$$

for $p \theta \geq 2, s, t \in[-1,1]$ with $s n^{1 / 2}, t n^{1 / 2} \in B_{0}^{n}(0)$.

Then, the hypercontractivity established in [33, Proposition 3.11, Proposition 3.12, and Proposition 3.16] allows us to estimate

$$
Q\left[\left|W_{\beta_{n}, T n}^{t n^{1 / 2}}(\eta)-W_{\beta_{n}, T n}^{s n^{1 / 2}}(\eta)\right|^{p \theta}\right] .
$$

Indeed,

$$
\begin{aligned}
& Q\left[\left|W_{\beta_{n}, T n}^{t n^{1 / 2}}(\eta)-W_{\beta_{n}, T n}^{s n^{1 / 2}}(\eta)\right|^{p \theta}\right]^{1 / p \theta} \\
& =Q\left[\left|\sum_{k=1}^{T n}\left(\Theta^{(k)}\left(t n^{1 / 2}\right)-\Theta^{(k)}\left(s n^{1 / 2}\right)\right)\right|^{p \theta}\right]^{1 / p \theta} \\
& \leq \sum_{k=1}^{T n} Q\left[\left|\Theta^{(k)}\left(t n^{1 / 2}\right)-\Theta^{(k)}\left(s n^{1 / 2}\right)\right|^{p \theta}\right]^{1 / p \theta} \\
& \leq \sum_{k=1}^{T n} \kappa_{p \theta}^{k}\left(Q\left[\left(\Theta^{(k)}\left(t n^{1 / 2}\right)-\Theta^{(k)}\left(s n^{1 / 2}\right)\right)^{2}\right]^{1 / 2}\right),
\end{aligned}
$$


where $\kappa_{p \theta}=2 \sqrt{p \theta-1} \sup _{n \geq 1} \frac{Q\left[e_{n}(0,0)^{p \theta}\right]^{1 / p \theta}}{Q\left[e_{n}(0,0)^{2}\right]^{1 / 2}}<\infty . \kappa_{p \theta}$ is finite since

$$
\lim _{n \rightarrow \infty} \frac{1}{\beta_{n}} Q\left[\left|e_{n}(0,0)\right|^{p \theta}\right]^{1 / p \theta}=Q\left[|\eta(0,0)|^{p \theta}\right]^{1 / p \theta} .
$$

We obtain from (3.15)

$$
\begin{aligned}
Q\left[\left|W_{\beta_{n}, T n}^{t n^{1 / 2}}(\eta)-W_{\beta_{n}, T n}^{s n^{1 / 2}}(\eta)\right|^{p \theta}\right] & \leq \\
& \leq C_{T, p, \theta}|t-s|^{\frac{p \theta}{2}}
\end{aligned}
$$

where we have used the fact that there exists a constant $c, C \in(0, \infty)$ such that for $x \geq \frac{1}{4}$

$$
c 2^{2 x} x^{1 / 2} \Gamma(x)^{2} \leq \Gamma(2 x) \leq C 2^{2 x} x^{1 / 2} \Gamma(x)^{2}
$$

which is a conclusion of the Stirling's formula [5, (3.9)]

$$
\Gamma(x)=\sqrt{2 \pi} x^{x-\frac{1}{2}} e^{-x+\frac{\theta_{x}}{12 x}}, \text { as } x \rightarrow \infty, \theta_{x} \in(0,1) .
$$

Thus, we find that for $p \geq \frac{2}{\theta}$

$$
\eta_{p, \theta}=\frac{p \theta}{2}
$$

in (3.11). Therefore, the proof completed when we take $p=\frac{5}{\theta}$ and $q=\frac{2 \theta}{3}$.

\subsection{Proof of Lemma 2.4}

The idea is the same as the proof of Lemma 2.3.

Proof of Lemma 2.4. For $x \in B_{0}(0)$, we set

$$
W_{\beta, T n}^{x}(\eta, A)=\sum_{y \in A} P_{S}^{x}\left[\prod_{k=1}^{T n} \zeta_{k, S_{k}}(\beta, \eta): S_{T n}=y\right]
$$

for $A \subset \mathbb{Z}$ and also, we regard $\left\{f_{n, A}(u)=W_{\beta, T n}^{u n^{1 / 2}}(\eta, A): u \in[-1,1]\right\}$ as a continuous function on $[-1,1]$ in a similar manner to $f_{n}(u)$.

It follows by the same argument as the proof of Lemma 2.3 that for $p=\frac{5}{\theta}, q=\frac{2 \theta}{3}$, and $z \in \mathbb{Z}$

$$
\begin{aligned}
Q\left[\max _{x \in B_{0}^{n}}\left(\sum_{w \in B_{z}^{n}} W_{\beta_{n}, T n}^{x}(\eta, w)\right)^{\theta}\right] & \leq Q\left[\left(W_{\beta_{n}, T n}\left(\eta, B_{z}^{n}\right)\right)^{\theta}\right]+C_{p, q} Q\left[B_{p, q, n, \theta, z, T}\right] \\
& \leq\left(\sum_{y \in B_{z}^{n}} p_{T n}(y)\right)^{\theta}+C_{p, q} Q\left[B_{p, q, n, \theta, z, T}\right]
\end{aligned}
$$

where

$$
B_{p, q, n, \theta, z, T}=\left(\int_{-1}^{1} \int_{-1}^{1} \frac{\left|f_{n, B_{z}^{n}}(u)^{\theta}-f_{n, B_{z}^{n}}(v)^{\theta}\right|^{p}}{|u-v|^{p q}} d u d v\right)^{1 / p}
$$


whose expectation is finite (see Proposition 3.4 below).

Since

$$
\sum_{w \in B_{z}^{n}} p_{T n}(x, w) \leq P\left(S_{T_{n}} \geq|2| z|-2| n^{1 / 2}\right) \leq \exp \left(-\frac{(2|z|-2)^{2}}{T}\right)
$$

for $x \in B_{0}(0)$ and for $|z| \geq T^{2}$, we have that

$$
\sum_{|z| \geq T^{2}}\left(\sum_{y \in B_{z}^{n}} p_{T n}(y)\right)^{\theta} \leq C_{\theta, 3} \exp \left(-C_{\theta, 4} T^{3}\right) .
$$

Combining this with Proposition 3.4, the proof has been completed.

Proposition 3.4. For $p=\frac{5}{\theta}>1$ and $\eta_{p, \theta}^{\prime}=\frac{9}{2}$, there exist $C_{\theta, 5}>0, C_{\theta, 6}>0$ such that

$$
Q\left[\left|f_{n, B_{z}^{n}}(u)^{\theta}-f_{n, B_{z}^{n}}(v)^{\theta}\right|^{p}\right] \leq C_{\theta, 5}|u-v|^{\eta_{p, \theta}^{\prime}} \exp \left(-\frac{C_{\theta, 6}|z|^{2}}{T}\right)
$$

for $u, v \in[-1,1]$.

In particular, for $p=\frac{5}{\theta}>1, q=\frac{2 \theta}{3}>0$, there exist $C_{\theta, 7}>0$ and $C_{\theta, 8}>0$ such that

$$
Q\left[B_{p, q, n, \theta, z, T}\right] \leq C_{\theta, 7} \exp \left(-\frac{C_{\theta, 8}|z|^{2}}{T}\right) .
$$

Proof of Proposition 3.4. We know that

$$
\left|f_{n, B_{z}^{n}}(u)^{\theta}-f_{n, B_{z}^{n}}(v)^{\theta}\right| \leq\left|W_{\beta_{n}, T n}^{u n^{1 / 2}}\left(\eta, B_{z}^{n}\right)-W_{\beta_{n}, T n}^{v n^{1 / 2}}\left(\eta, B_{z}^{n}\right)\right|^{\theta} .
$$

as the proof of Proposition 3.3. In the following, we will estimate $Q\left[\left|W_{\beta_{n}, T n}^{u n^{1 / 2}}\left(\eta, B_{z}^{n}\right)-W_{\beta_{n}, T n}^{v n^{1 / 2}}\left(\eta, B_{z}^{n}\right)\right|^{p \theta}\right]$.

We write

$$
\begin{aligned}
& W_{\beta_{n}, T n}^{x}(\eta, w) \\
& =P_{S}^{x}\left[\prod_{i=1}^{T n}\left(1+e_{n}\left(i, S_{i}\right)\right): S_{T n}=w\right] \\
& =p_{T n}(w-x) \\
& +\sum_{k=1}^{T n} \sum_{1 \leq i_{1}<\cdots<i_{k} \leq T n} \sum_{\mathbf{x} \in \mathbb{Z}^{k}}\left(\prod_{i=1}^{k} p_{i_{j}-i_{j-1}}\left(x_{i}-x_{j-1}\right) e_{n}\left(i_{j}, x_{j}\right)\right) p_{T n-i_{k}}\left(w-x_{k}\right) \\
& =\sum_{k=0}^{T n} \Theta^{(k)}(x, w),
\end{aligned}
$$

where

$$
\begin{aligned}
& \Theta^{(k)}(x, w) \\
& = \begin{cases}p_{T n}(x, w), & k=0 \\
\sum_{k=1}^{T n} \sum_{1 \leq i_{1}<\cdots<i_{k} \leq T n} \sum_{\mathbf{x} \in \mathbb{Z}^{k}}\left(\prod_{i=1}^{k} p_{i_{j}-i_{j-1}}\left(x_{i}-x_{j-1}\right) e_{n}\left(i_{j}, x_{j}\right)\right) p_{T n-i_{k}}\left(w-x_{k}\right), & k \geq 1 .\end{cases}
\end{aligned}
$$


where we set $i_{0}=0$ and $x_{0}=x$.

Then, we have that

$$
\begin{aligned}
& Q\left[\Theta^{(k)}(x, w)\right]=0, \quad k \geq 1 \\
& Q\left[\Theta^{(k)}(x, y) \Theta^{(\ell)}(z, w)\right]=0, \quad k \neq \ell .
\end{aligned}
$$

Hence,

$$
\begin{aligned}
& \left(\sum_{w \in B_{z}^{n}}\left(\Theta^{(0)}(x, w)-\Theta^{(0)}(y, w)\right)\right)^{2} \\
& =\left(\sum_{w \in B_{z}^{n}}\left(p_{T n}(w-x)-p_{T n}(w-y)\right)\right)^{2} \\
& \leq\left(\sum_{w \in B_{z}^{n}}\left|p_{T n}(w-x)-p_{T n}(w-y)\right|\right)\left(\sum_{w \in B_{z}^{n}}\left(p_{T n}(w-x)+p_{T n}(w-y)\right)\right)
\end{aligned}
$$

and (3.13) implies that for $u, v \in[-1,1]$ with $v<u$ and for $z \geq T^{2}$

$$
\begin{aligned}
& \sum_{w \in B_{z}^{n}}\left|p_{T n}\left(w-u n^{1 / 2}\right)-p_{T n}\left(w-v n^{1 / 2}\right)\right| \\
& \leq \sum_{w \in B_{z}^{n}} \frac{c|u-v| n^{1 / 2}}{(T n)^{3 / 2}} \\
& +\sum_{w \in B_{z}^{n}} \frac{2}{\sqrt{2 \pi T n}} \exp \left(-\frac{\left(u n^{1 / 2}-w\right)^{2}}{2 T n}\right)\left(1-\exp \left(-\frac{(u-v)\left(2 w-u n^{1 / 2}-v n^{1 / 2}\right)}{2 T n^{1 / 2}}\right)\right) \\
& \leq c \frac{|u-v|}{T^{3 / 2} n^{1 / 2}}+\frac{2}{\sqrt{2 \pi T n}} \sum_{w \in B_{z}^{n}} \exp \left(-\frac{\left(u n^{1 / 2}-w\right)^{2}}{2 T n}\right) \frac{(u-v)\left(2 w-u n^{1 / 2}-v n^{1 / 2}\right)}{2 T n^{1 / 2}} \\
& \leq c \frac{|u-v|}{T^{3 / 2} n^{1 / 2}}+\frac{4|u-v|}{\sqrt{2 \pi T}} \exp \left(-\frac{|z-1|^{2}}{2 T}\right) \frac{2 z+4}{2 T} \\
& =C_{T, 2}|u-v|,
\end{aligned}
$$

where $C_{T, 2} \rightarrow 0$ as $T \rightarrow \infty$. The same argument holds for $u<v$ or for $z \leq-T^{2}$. Combining this with (3.17), we have that for $u, v \in[-1,1]$ and $|z| \geq T^{2}$

$$
\begin{aligned}
& \left(\sum_{w \in B_{z}^{n}}\left(\Theta^{(0)}\left(u n^{\frac{1}{2}}, w\right)-\Theta^{(0)}\left(v n^{\frac{1}{2}}, w\right)\right)\right)^{2} \\
& \leq C_{T, 2}|u-v|\left(\sum_{w \in B_{z}^{n}}\left(p_{T n}\left(w-u n^{\frac{1}{2}}\right)+p_{T n}\left(w-v n^{\frac{1}{2}}\right)\right)\right) \\
& \leq 2 C_{T, 2}|u-v| \exp \left(-\frac{(2|z|-2)^{2}}{T}\right) .
\end{aligned}
$$


Also, we have that for $k \geq 1$

$$
\begin{aligned}
& Q\left[\left(\sum_{w \in B_{z}^{n}}\left(\Theta^{(k)}\left(u n^{\frac{1}{2}}, w\right)-\Theta^{(k)}\left(v n^{\frac{1}{2}}, w\right)\right)\right)^{2}\right] \\
& \left.=Q\left[e_{n}(0,0)^{2}\right]^{k} \sum_{1 \leq i_{1}<\cdots<i_{k} \leq T n} \sum_{\mathbf{x} \in \mathbb{Z}^{k}} \sum \sum^{2} p_{i_{1}}\left(x_{1}-u n^{\frac{1}{2}}\right)-p_{i_{1}}\left(x_{1}-v n^{\frac{1}{2}}\right)\right)^{2}\left(\prod_{i=2}^{k} p_{i_{j}-i_{j-1}}\left(x_{j}-x_{j-1}\right)^{2}\right)\left(\sum_{w \in B_{z}^{n}} p_{T n-i_{k}}\left(w-x_{k}\right)\right)^{2} \\
& \leq Q\left[e_{n}(0,0)^{2}\right]^{k} \sum_{1 \leq i_{1}<\cdots<i_{k} \leq T n}\left(p_{2 i_{1}}(0)-p_{2 i_{1}}\left(n^{\frac{1}{2}}(u-v)\right)\right) \prod_{i=2}^{k} p_{2\left(i_{j}-i_{j-1}\right)}(0) \\
& \leq C|u-v| \frac{C_{5}^{k-1} T^{\frac{k-1}{2}}}{\Gamma\left(\frac{k-1}{2}+1\right)}
\end{aligned}
$$

as the proof of Lemma 2.3 .

Hence, we obtain by Hölder's inequality that for $p=\frac{5}{\theta}$

$$
\begin{aligned}
& Q\left[\left|W_{\beta_{n}, T n}^{u n}\left(\eta, B_{z}^{n}\right)-W_{\beta_{n}, T n}^{v n^{\frac{1}{2}}}\left(\eta, B_{z}^{n}\right)\right|^{p \theta}\right] \\
& \leq Q\left[\left|W_{\beta_{n}, T n}^{u n^{\frac{1}{2}}}\left(\eta, B_{z}^{n}\right)-W_{\beta_{n}, T n}^{v n^{\frac{1}{2}}}\left(\eta, B_{z}^{n}\right)\right|^{\frac{9}{2}}\left|W_{\beta_{n}, T n}^{u n^{\frac{1}{2}}}\left(\eta, B_{z}^{n}\right)+W_{\beta_{n}, T n}^{v n^{\frac{1}{2}}}\left(\eta, B_{z}^{n}\right)\right|^{\frac{1}{2}}\right] \\
& \leq Q\left[\left|W_{\beta_{n}, T n}^{u n^{\frac{1}{2}}}\left(\eta, B_{z}^{n}\right)-W_{\beta_{n}, T n}^{v n^{\frac{1}{2}}}\left(\eta, B_{z}^{n}\right)\right|^{9}\right]^{\frac{1}{2}} Q\left[W_{\beta_{n}, T n}^{u n^{\frac{1}{2}}}\left(\eta, B_{z}^{n}\right)+W_{\beta_{n}, T n}^{v n^{\frac{1}{2}}}\left(\eta, B_{z}^{n}\right)\right]^{\frac{1}{2}} \\
& \leq C_{T, 3}|u-v|^{9 / 2}\left(\sum_{w \in B_{z}^{n}}\left(p_{T n}\left(u n^{\frac{1}{2}}, w\right)+p_{T n}\left(v n^{\frac{1}{2}}, w\right)\right)\right)^{\frac{1}{2}} \\
& \leq \sqrt{2} C_{T, 3}|u-v|^{9 / 2} \exp \left(-\frac{2|| z|-1|^{2}}{T}\right)
\end{aligned}
$$

where we have used the hypercontractivity as the proof of Lemma 2.3. In particular, $C_{T, 3}$ is given by

$$
C\left(\sum_{k \geq 1} \kappa_{9}^{k}\left(\frac{C_{5}^{k-1} T^{\frac{k-1}{2}}}{\Gamma\left(\frac{k-1}{2}+1\right)}\right)^{1 / 2}\right)^{9}
$$

and we can find from (3.16) that $C_{T, 3}$ is independent of the choice of $z$ and

$$
\varlimsup_{T \rightarrow \infty} \frac{1}{T} \log C_{T, 3} \leq C<\infty
$$

Thus, we have that there exist $C_{\theta, 5}>0$ and $C_{\theta, 6}>0$

$$
Q\left[\left|f_{n, B_{z}^{n}}(u)^{\theta}-f_{n, B_{z}^{n}}(v)^{\theta}\right|^{p}\right] \leq C_{\theta, 5}|u-v|^{9 / 2} \exp \left(-\frac{C_{\theta, 6}|z|^{2}}{T}\right)
$$

for $u, v \in[-1,1]$. 
Also, when we take $p=\frac{5}{\theta}>1, q=\frac{2 \theta}{3}>0$ with $p q=\frac{10}{3}>2$ in (3.8), there exist $C_{\theta, 7}>0, C_{\theta, 8}>0$ and $\eta_{p, \theta}^{\prime}=\frac{9}{2}$ which satisfies $\eta_{p, \theta}^{\prime}-p q=\frac{7}{6}>-1$ so that

$$
\begin{aligned}
Q\left[B_{p, q, n, \theta, z, T}\right] & \leq Q\left[B_{p, q, n, \theta, z, T}^{p}\right]^{1 / p} \\
& =\left(\int_{-1}^{1} \int_{-1}^{1} \frac{Q\left[\left|W_{\beta_{n}, T n}^{u n^{\frac{1}{2}}}\left(\eta, B_{z}^{n}\right)-W_{\beta_{n}, T n}^{v n^{\frac{1}{2}},}\left(\eta, B_{z}^{n}\right)\right|^{p \theta}\right]}{|u-v|^{p q}} d u d v\right)^{1 / p} \\
& \leq C_{\theta, 7} \exp \left(-\frac{C_{\theta, 8}|z|^{2}}{T}\right) .
\end{aligned}
$$

\section{Continuum directed polymers}

To prove Lemma 2.2 and Lemma 2.5, we recall the property of continuum directed polymers.

\subsection{Continuum directed polymers}

The mild solution to stochastic heat equation

$$
\partial_{t} \mathcal{Z}=\frac{1}{2} \Delta \mathcal{Z}+\beta \mathcal{Z} \dot{\mathcal{W}}, \lim _{t \searrow 0} \mathcal{Z}(t, y) d y=\delta_{x}(d y)
$$

has the following representation using Wiener chaos expansion: For $x, y \in \mathbb{R}$

$$
\begin{aligned}
\mathcal{Z}_{\beta}^{x}(T, y) & =\rho_{T}(x, y) \\
& +\sum_{n \geq 1} \beta^{n} \int_{\Delta_{n}(0, T)} \int_{\mathbb{R}^{n}} \boldsymbol{\rho}_{n}(\mathbf{t}, \mathbf{x} \mid 0, x ; T, y) \mathcal{W}\left(d t_{1} d x_{1}\right) \cdots \mathcal{W}\left(d t_{n} d x_{n}\right),
\end{aligned}
$$

where we set

$$
\rho_{t}(x, y)=\rho_{t}(x-y)=\frac{1}{\sqrt{2 \pi t}} \exp \left(-\frac{(x-y)^{2}}{2 t}\right), \quad t>0, x, y \in \mathbb{R},
$$

and

$$
\begin{aligned}
\boldsymbol{\rho}_{1}\left(t_{1}, x_{1} \mid s, x ; t, y\right) & =\rho_{t_{1}-s}\left(x, x_{1}\right) \rho_{t-t_{1}}\left(x_{1}, y\right) \\
\boldsymbol{\rho}_{n}(\mathbf{t}, \mathbf{x} \mid s, x ; t, y) & =\rho_{t_{1}-s}\left(x, x_{1}\right)\left(\prod_{i=1}^{n-1} \rho_{t_{i+1}-t_{i}}\left(x_{i}, x_{i+1}\right)\right) \rho_{t-t_{n}}\left(x_{n}, y\right)
\end{aligned}
$$

for $x, y \in \mathbb{R}, \mathbf{x}=\left(x_{1}, \cdots, x_{n}\right) \in \mathbb{R}^{n}, 0 \leq s<t$ and for

$$
\mathbf{t} \in \Delta_{n}(s, t)=\left\{\mathbf{t}=\left(t_{1}, \cdots, t_{n}\right): s<t_{1}<\cdots<t_{n}<t\right\} .
$$

Also, we define the four parameter field by

$$
\begin{aligned}
& \mathcal{Z}_{\beta}(s, x ; t, y)=\rho_{t-s}(x, y) \\
& \quad+\sum_{n \geq 1} \beta^{n} \int_{\Delta_{n}(s, t)} \int_{\mathbb{R}^{n}} \boldsymbol{\rho}_{n}(\mathbf{t}, \mathbf{x} \mid s, x ; t, y) \mathcal{W}\left(d t_{1} d x_{1}\right) \cdots \mathcal{W}\left(d t_{n} d x_{n}\right),
\end{aligned}
$$


for $0 \leq s<t<\infty$ and for $x, y \in \mathbb{R}$.

Also, we define

$$
\mathcal{Z}_{\beta}^{(s, x)}(t)=\int_{\mathbb{R}} \mathcal{Z}_{\beta}(s, x ; t, y) d y, \text { for } 0 \leq s<t<\infty, \quad x \in \mathbb{R} .
$$

We write

$$
\mathcal{Z}_{\beta}(t, x)=\mathcal{Z}_{\beta}(0,0 ; t, x), \mathcal{Z}_{\beta}(t)=\mathcal{Z}_{\beta}^{(0,0)}(t), \text { and } \mathcal{Z}_{\beta}^{x}=\mathcal{Z}_{\beta}^{(0, x)}(t)
$$

for $t>0$ and $x \in \mathbb{R}$.

We denote by $P_{\mathcal{Z}}$ the probability measure on the white noise $\mathcal{W}$ on $[0, \infty) \times \mathbb{R}$.

Then, we have the following fact [1, Theorem 3.1]:

Theorem 4.1. There exists a version of the field $\mathcal{Z}_{\beta}(s, x ; t, y)$ which is jointly continuous in all four variables and have the following properties:

(i) $P_{\mathcal{Z}}\left[\mathcal{Z}_{\beta}(s, x ; t, y)\right]=\rho_{t-s}(y-x)$.

(ii) (Stationary): $\mathcal{Z}_{\beta}(s, x ; t, y) \stackrel{d}{=} \mathcal{Z}_{\beta}\left(s+u_{0}, x+z_{0} ; t+u_{0}, y+z_{0}\right)$.

(iii) (Scaling): $\mathcal{Z}_{\beta}\left(r^{2} s, r x ; r^{2} t, r y\right) \stackrel{d}{=} \frac{1}{r} \mathcal{Z}_{\beta \sqrt{r}}(s, x ; t, y)$.

(iv) (Positivity): With probability one, $\mathcal{Z}_{\beta}(s, x ; t, y)$ is strictly positive for all tuples $(s, x ; t, y)$ with $0 \leq s<t$.

(v) The law of $\frac{\mathcal{Z}_{\beta}(s, x ; t, y)}{\rho_{t-s}(y-x)}$ does not depend on $x$ or $y$.

(vi) It has an independent property among disjoint time intervals: for any finite $\left\{\left(s_{1}, t_{i}\right]\right\}_{i=1}^{n}$ and any $x_{i}, y_{i} \in \mathbb{R}$, the random variables $\left\{\mathcal{Z}_{\beta}\left(s_{i}, x_{i} ; t_{i}, y_{i}\right)\right\}_{i=1}^{n}$ are mutually independent.

(vii) (Chapman-Kolmogorov equations): With probability one, for all $0 \leq s<r<t$ and $x, y \in \mathbb{R}$,

$$
\mathcal{Z}_{\beta}(s, x ; t, y)=\int_{\mathbb{R}} \mathcal{Z}_{\beta}(s, x ; r, z) \mathcal{Z}_{\beta}(r, z ; t, y) d z .
$$

We remark that by Fubini's theorem with Theorem 4.1 (i) and (iv)

$$
P_{\mathcal{Z}}\left[\mathcal{Z}_{\beta}(t)\right]=1
$$

for $t>0$. Also, Theorem 4.1 (iii) implies that for any $t>0, x \in \mathbb{R}, \beta \in \mathbb{R}, r>0$

$$
\frac{\mathcal{Z}_{\beta}\left(0,0, r^{2} t, r x\right)}{\rho_{r^{2} t}(r x)} \stackrel{d}{=} \frac{\mathcal{Z}_{\beta \sqrt{r}}(0,0, t, x)}{\rho_{t}(x)}
$$

The following is a corollary of [4, Theorem 1.1].

Theorem 4.2. $\frac{1}{T} \log \mathcal{Z}_{1}(T, 0)$ converges to $-\frac{1}{4 !}$ in probability as $T \rightarrow \infty$.

Also, the following is the result obtained by Moreno [32]:

Corollary 4.3. For any $\beta \geq 0, t>0, x \in \mathbb{R}$ and $p \geq 1$, we have that $\left(\mathcal{Z}_{\sqrt{2}}(t)\right)^{-1} \in L^{p}$ and $\left(\mathcal{Z}_{\sqrt{2}}(t, x)\right)^{-1} \in L^{p}$. 


\subsection{Proof of Lemma 2.2 and Lemma 2.5}

We can apply some techniques of discrete directed polymers in random environment to continuum directed polymer. The proof of the following lemma is such an example. Actually, the proof is analogous to the concentration inequality of DPRE [19, Proposition 1.5] and [31].

Lemma 4.4. We have that for $\beta>0$

$$
\varlimsup_{\theta \rightarrow 0} \varlimsup_{T \rightarrow \infty} \frac{1}{T \theta} \log P_{\mathcal{Z}}\left[\left(\mathcal{Z}_{\beta}(T)\right)^{\theta}\right] \leq F_{\mathcal{Z}}(\beta) .
$$

To prove Lemma 4.4, we use the following lemma.

Lemma 4.5. ([31, Theorem 2.1]) Let $(\Omega, \mathcal{F}, P)$ be a probability space. Let $\left\{X_{i}: 1 \leq i \leq\right.$ $n\}$ be a finite sequence of supermartingale differences with filtration $\mathcal{F}_{i}$ with $\mathcal{F}_{0}=\{\emptyset, \Omega\}$. If for some constant $K>0$ and all $i \in\{1, \cdots, n\}$,

$$
P\left[e^{\left|X_{i}\right|} \mid \mathcal{F}_{i-1}\right] \leq K, \quad \text { a.s., }
$$

then

$$
P\left[e^{t S_{n}}\right] \leq \exp \left(\frac{t^{2} K n}{1-t}\right), \quad \text { for all } t \in(0,1)
$$

and

$$
P\left(\frac{S_{n}}{n}>x\right) \leq \exp \left(-(\sqrt{x+K}-\sqrt{K})^{2}\right), \text { for all } x>0,
$$

where $S_{0}=0$ and for $i \in\{1, \cdots, n\}, S_{i}=S_{i-1}+X_{i}$.

Proof of Lemma 4.4. It is enough to show that there exists a $K=K_{\beta}>0$ such that

$$
P_{\mathcal{Z}}\left[\exp \left(\theta\left(\log \mathcal{Z}_{\beta}(T)-P_{\mathcal{Z}}\left[\log \mathcal{Z}_{\beta}(T)\right]\right)\right)\right] \leq \exp \left(\frac{T \theta^{2} K}{1-|\theta|}\right)
$$

for $|\theta| \in(0,1)$.

We define $\sigma$-field

$$
\begin{aligned}
& \mathcal{F}_{i}=\sigma[\mathcal{W}(t, x): 0 \leq t \leq i, x \in \mathbb{R}] \\
& \tilde{\mathcal{F}}_{i}=\sigma[\mathcal{W}(t, x): t \notin[i-1, i], x \in \mathbb{R}]
\end{aligned}
$$

Then, we write

$$
\log \mathcal{Z}_{\beta}(T)-P_{\mathcal{Z}}\left[\log \mathcal{Z}_{\beta}(T)\right]=\sum_{i=1}^{T} V_{i}^{T}
$$

where

$$
V_{i}^{T}=P_{\mathcal{Z}}\left[\log \mathcal{Z}_{\beta}(T) \mid \mathcal{F}_{i}\right]-P_{\mathcal{Z}}\left[\log \mathcal{Z}_{\beta}(T) \mid \mathcal{F}_{i-1}\right]
$$

are martingale differences. Thus, it is enough to check (4.2) for $\left\{V_{i}^{T}: 1 \leq i \leq T\right\}$. Here, we introduce new random variables

$$
\begin{aligned}
\hat{\mathcal{Z}}_{\beta}(i, T) & =P_{\mathcal{Z}}\left[\mathcal{Z}_{\beta}(T) \mid \tilde{\mathcal{F}}_{i}\right] \\
& =\int_{\mathbb{R}^{2}} \mathcal{Z}_{\beta}(i-1, x) \rho_{1}(x, y) \mathcal{Z}_{\beta}^{(i, y)}(T) d x d y .
\end{aligned}
$$


Since it is clear that

$$
P_{\mathcal{Z}}\left[\log \hat{\mathcal{Z}}_{\beta}(i, T) \mid \mathcal{F}_{i-1}\right]=P_{\mathcal{Z}}\left[\log \hat{\mathcal{Z}}_{\beta}(i, T) \mid \mathcal{F}_{i}\right]
$$

we have

$$
V_{i}^{T}=P_{\mathcal{Z}}\left[\log \frac{\mathcal{Z}_{\beta}(T)}{\hat{\mathcal{Z}}_{\beta}(i, T)} \mid \mathcal{F}_{i}\right]-P_{\mathcal{Z}}\left[\log \frac{\mathcal{Z}_{\beta}(T)}{\hat{\mathcal{Z}}_{\beta}(i, T)} \mid \mathcal{F}_{i-1}\right]
$$

Also, we consider a new probability measure on $\mathbb{R}^{2}$ by

$$
\begin{aligned}
& \mu_{T}^{(i)}(x, y) d x d y \\
& =\frac{1}{\hat{\mathcal{Z}}_{\beta}(i, T)} \mathcal{Z}_{\beta}(i-1, x) \rho_{1}(x, y) \mathcal{Z}_{\beta}^{(i, y)}(T) d x d y
\end{aligned}
$$

We should remark that $\left\{\mu_{T}^{(i)}(x, y):(x, y) \in \mathbb{R}^{2}\right\}$ is $\tilde{\mathcal{F}}_{i}$-measurable.

Then, it is clear that

$$
\frac{\mathcal{Z}_{\beta}(T)}{\hat{\mathcal{Z}}_{\beta}(i, T)}=\int_{\mathbb{R}^{2}} \frac{\mathcal{Z}_{\beta}(i-1, x ; i, y)}{\rho_{1}(x, y)} \mu_{T}^{(i)}(x, y) d x d y
$$

and

$$
P_{\mathcal{Z}}\left[\frac{\mathcal{Z}_{\beta}(T)}{\hat{\mathcal{Z}}_{\beta}(i, T)} \mid \mathcal{F}_{i-1}\right]=P_{\mathcal{Z}}\left[P_{\mathcal{Z}}\left[\frac{\mathcal{Z}_{\beta}(T)}{\hat{\mathcal{Z}}_{\beta}(i, T)} \mid \tilde{\mathcal{F}}_{i}\right] \mid \mathcal{F}_{i-1}\right]=1 .
$$

Thus, Jensen's inequality implies from Theorem 4.1 (ii) and (iv) that

$$
\begin{aligned}
0 \leq-P_{\mathcal{Z}}\left[\log \frac{\mathcal{Z}_{\beta}(T)}{\hat{\mathcal{Z}}_{\beta}(i, T)} \mid \mathcal{F}_{i-1}\right] & \leq-P_{\mathcal{Z}}\left[\log \frac{\mathcal{Z}_{\beta}(0,0 ; 1,0)}{p_{1}(0)}\right] \\
& \leq C_{6}
\end{aligned}
$$

where we have used that

$$
-P_{\mathcal{Z}}\left[\log \frac{\mathcal{Z}_{\beta}(0,0 ; 1,0)}{p_{t}(0)}\right] \leq C_{6}
$$

(see Corollary 4.3).

Thus, we have from Jensen's inequality that

$$
P_{\mathcal{Z}}\left[\exp \left(V_{i}(T)\right) \mid \mathcal{F}_{i-1}\right] \leq e^{C_{6}} P_{\mathcal{Z}}\left[P_{\mathcal{Z}}\left[\frac{\mathcal{Z}_{\beta}(T)}{\hat{\mathcal{Z}}_{\beta}(i, T)} \mid \mathcal{F}_{i}\right] \mid \mathcal{F}_{i-1}\right]=e^{C_{6}} .
$$

Also, Jensen's inequality implies that

$$
\begin{aligned}
& P_{\mathcal{Z}}\left[\exp \left(-V_{i}(T)\right) \mid \mathcal{F}_{i-1}\right] \leq P_{\mathcal{Z}}\left[P_{\mathcal{Z}}\left[\frac{\hat{\mathcal{Z}}_{\beta}(i, T)}{\mathcal{Z}_{\beta}(T)} \mid \mathcal{F}_{i}\right] \mid \mathcal{F}_{i-1}\right] \\
& \leq P_{\mathcal{Z}}\left[P_{\mathcal{Z}}\left[\int_{\mathbb{R}^{2}}\left(\frac{\mathcal{Z}_{\beta}(i-1, x ; i, y)}{\rho_{1}(x, y)}\right)^{-1} \mu_{T}^{(i)}(x, y) d x d y \mid \mathcal{F}_{i}\right] \mid \mathcal{F}_{i-1}\right] \leq C_{7},
\end{aligned}
$$

where we have used that

$$
P_{\mathcal{Z}}\left[\left(\frac{\mathcal{Z}_{\beta}(0, x ; 1, y)}{\rho_{1}(x, y)}\right)^{-1}\right] \leq C_{7}
$$

Thus, we have confirmed (4.2) so that we have proved (4.3). 
We can find that the above proof is true when we replace $\mathcal{Z}_{\beta}(T)$ by $\mathcal{Z}_{\beta}(T, 0)$. Therefore, we have the following corollary from (4.3).

\section{Corollary 4.6. We have}

$$
\lim _{T \rightarrow \infty} \frac{1}{T} P_{\mathcal{Z}}\left[\left|\log \mathcal{Z}_{\beta}(T)-P_{\mathcal{Z}}\left[\log \mathcal{Z}_{\beta}(T)\right]\right|\right]=0
$$

and

$$
\lim _{T \rightarrow \infty} \frac{1}{T} P_{\mathcal{Z}}\left[\left|\log \mathcal{Z}_{\beta}(T, 0)-P_{\mathcal{Z}}\left[\log \mathcal{Z}_{\beta}(T, 0)\right]\right|\right]=0 .
$$

In particular, we have from (4.1) and Theorem 4.2 that

$$
\lim _{T \rightarrow \infty} \frac{1}{T} \log P_{\mathcal{Z}}\left[\mathcal{Z}_{\beta}(T, 0)\right]=-\frac{\beta^{4}}{24} .
$$

Now, we can prove a part of Lemma 2.2.

Proof of (2.2) in Lemma 2.2. Here, we prove the existence of the limit $F_{\mathcal{Z}}(\beta)$ in Lemma 2.2 and give the lower bound. The upper bound will be given in the end of this subsection.

We should remark that the integrability of $\log \mathcal{Z}_{\beta}(t)$ follows from Theorem 4.1 (i) and Corollary 4.3. Then, we have that for any $s, t>0$

$$
\begin{aligned}
P_{\mathcal{Z}}\left[\log \mathcal{Z}_{\beta}(s+t)\right]= & P_{\mathcal{Z}}\left[\log \mathcal{Z}_{\beta}(s)\right] \\
& \quad+P_{\mathcal{Z}}\left[\log \int_{\mathbb{R}} \frac{\mathcal{Z}_{\beta}(s, x)}{\mathcal{Z}_{\beta}(s)} \mathcal{Z}_{\beta}^{(s, x)}(s+t) d x\right] \\
\geq & P_{\mathcal{Z}}\left[\log \mathcal{Z}_{\beta}(s)\right]+P_{\mathcal{Z}}\left[\log \mathcal{Z}_{\beta}(t)\right]
\end{aligned}
$$

where we have used Theorem 4.1 (vii) in the first line and Jensen's inequality and Theorem 4.1 (ii) in the last line. Thus, for any $t>0,\left\{P_{\mathcal{Z}}\left[\log \mathcal{Z}_{\beta}(n t)\right]: n \in \mathbb{N}\right\}$ is superadditive and hence

$$
F_{\mathcal{Z}}^{(t)}(\beta)=\lim _{n \rightarrow \infty} \frac{1}{n t} P_{\mathcal{Z}}\left[\log \mathcal{Z}_{\beta}(n t)\right]=\sup _{n \geq 1} \frac{1}{n t} P_{\mathcal{Z}}\left[\log \mathcal{Z}_{\beta}(n t)\right]
$$

exists.

The independence of $F_{\mathcal{Z}}^{(t)}(\beta)$ in $t>0$ is easy. Fix $t>0$. Then, for any $T>t$, there exists $n \in \mathbb{N}$ such that $n t \leq T<(n+1) t$ and hence

$$
\begin{aligned}
& \frac{n t}{T} \frac{1}{n t}\left(P_{\mathcal{Z}}\left[\log \mathcal{Z}_{\beta}(n t)\right]+P_{\mathcal{Z}}\left[\log \mathcal{Z}_{\beta}(T-n t)\right]\right) \\
& \leq \frac{1}{T} P_{\mathcal{Z}}\left[\log \mathcal{Z}_{\beta}(T)\right] \\
& =\frac{1}{T}\left(P_{\mathcal{Z}}\left[\log \mathcal{Z}_{\beta}(n t)\right]\right. \\
& \left.\quad+P_{\mathcal{Z}}\left[\log \int_{\mathbb{R}} \frac{\mathcal{Z}_{\beta}(n t, x)}{\mathcal{Z}_{\beta}(n t)} \mathcal{Z}_{\beta}^{(n t, x)}(T) d x\right]\right) \\
& \leq \frac{n t}{T} \frac{1}{n t} P_{\mathcal{Z}}\left[\log \mathcal{Z}_{\beta}(n t)\right],
\end{aligned}
$$

where we have used Jensen's inequality and Theorem 4.1 (i) in the last line. Also, we know from Jensen's inequality and Theorem 4.1 (i) and (vii) that for any $s \in(0,1)$

$$
\begin{aligned}
P_{\mathcal{Z}}\left[\log \mathcal{Z}_{\beta}(1)\right] & =P_{\mathcal{Z}}\left[\log \mathcal{Z}_{\beta}(s)\right]+P_{\mathcal{Z}}\left[\log \int_{\mathbb{R}} \frac{\mathcal{Z}_{\beta}(s, x)}{\mathcal{Z}_{\beta}(s)} \mathcal{Z}_{\beta}^{(s, x)}(1) d x\right] \\
& \leq P_{\mathcal{Z}}\left[\log \mathcal{Z}_{\beta}(s)\right] \leq \log P_{\mathcal{Z}}\left[\mathcal{Z}_{\beta}(s)\right]=0 .
\end{aligned}
$$


Thus, taking limit in $T \rightarrow \infty$, we find the limit $F_{\mathcal{Z}}(\beta)=\lim _{T \rightarrow \infty} \frac{1}{T} P_{\mathcal{Z}}\left[\log \mathcal{Z}_{\beta}(T)\right]$ exists and that $F_{\mathcal{Z}}(\beta)=\sup _{T>0} \frac{1}{T} P_{\mathcal{Z}}\left[\log \mathcal{Z}_{\beta}(T)\right]$.

Remark 4.7. We are almost ready to give the proof of Lemma 2.5. The idea is similar to the proofs of Lemma 2.3 and Lemma 2.4. However, it is difficult to prove Lemma 2.5 by applying (3.7) to the continuous function $\left\{\mathcal{Z}_{\sqrt{2}}^{x}(T): x \in[-1,1]\right\}$ directly .

Indeed, if we apply (3.7) to $\left\{\mathcal{Z}_{\sqrt{2}}^{x}(T): x \in[-1,1]\right\}$ for $p \geq 1, q>0$ with $p q>2$, then we have

$$
\begin{aligned}
& P_{\mathcal{Z}}\left[\sup _{x \in[-1,1]} \mathcal{Z}_{\sqrt{2}}^{x}(T)^{\theta}\right] \\
& \leq P_{\mathcal{Z}}\left[\mathcal{Z}_{\sqrt{2}}(T)^{\theta}\right]+C_{p, q} P_{\mathcal{Z}}\left[\left(\int_{-1}^{1} \int_{-1}^{1} \frac{\left|\mathcal{Z}_{\sqrt{2}}^{x}(T)^{\theta}-\mathcal{Z}_{\sqrt{2}}^{y}(T)^{\theta}\right|^{p}}{|x-y|^{p q}} d x d y\right)^{1 / p}\right] .
\end{aligned}
$$

We know the first term in the right hand side decays exponentially from Lemma 4.4. The simplest way to estimate the second term in the right hand side is the second moment $P_{\mathcal{Z}}\left[\left(\mathcal{Z}_{\sqrt{2}}^{x}(T)-\mathcal{Z}_{\sqrt{2}}^{y}(T)\right)^{2}\right]$ which we can calculate

$$
\begin{aligned}
& P_{\mathcal{Z}}\left[\left(\mathcal{Z}_{\sqrt{2}}^{x}(T)-\mathcal{Z}_{\sqrt{2}}^{y}(T)\right)^{2}\right] \\
& =\sum_{n \geq 1} 2^{\frac{n}{2}} \int_{\Delta_{n}(0, T)} \int_{\mathbb{R}^{n}}\left(\int_{\mathbb{R}}\left(\boldsymbol{\rho}_{n}(\mathbf{t}, \mathbf{x} \mid 0, x ; T, w)-\boldsymbol{\rho}_{n}(\mathbf{t}, \mathbf{x} \mid 0, y ; T, w)\right) d w\right)^{2} d \mathbf{t} \mathbf{x} \\
& =\sum_{n \geq 1} 2^{\frac{n}{2}} \int_{\Delta_{n}(0, T)} \int_{\mathbb{R}^{n}}\left(\rho_{t_{1}}\left(x, x_{1}\right)-\rho_{t_{1}}\left(y, x_{1}\right)\right)^{2} \prod_{i=1}^{n} \rho_{t_{i}-t_{i-1}}\left(x_{i-1}, x_{i}\right)^{2} d \mathbf{t} d \mathbf{x} .
\end{aligned}
$$

From (A.5), (A.7), and (A.10), it is bounded from above by

$$
\sum_{n \geq 1} C_{8} \frac{|x-y| T^{\frac{n-1}{2}}}{2^{\frac{n-2}{2}} \Gamma\left(\frac{n+1}{2}\right)}
$$

which diverges exponentially in $T$.

In the following proofs of Lemmas, we will write only the important parts and postpone the bothersome parts with hard calculations as the propositions to section 5 .

Proof of Lemma 2.5. We write

$$
\begin{aligned}
\mathcal{Z}_{\sqrt{2}}^{x}(T) & =\int_{\mathbb{R}} \mathcal{Z}_{\sqrt{2}}^{x}(1, w) \mathcal{Z}_{\sqrt{2}}^{(1, w)}(T) d w \\
& =\int_{A(T)} \mathcal{Z}_{\sqrt{2}}^{x}(1, w) \mathcal{Z}_{\sqrt{2}}^{(1, w)}(T) d w+\int_{A(T)^{c}} \mathcal{Z}_{\sqrt{2}}^{x}(1, w) \mathcal{Z}_{\sqrt{2}}^{(1, w)}(T) d w \\
& =: I_{1}(T, x)+I_{2}(T, x),
\end{aligned}
$$

where $A(T)=[-2 T-1,2 T+1]$. Hereafter, we will look at $I_{1}(T, x)$ and $I_{2}(T, x)$.

We will show in the lemmas below that

$$
\varlimsup_{\theta \rightarrow 0} \varlimsup_{T \rightarrow \infty} \frac{1}{\theta T} \log P_{\mathcal{Z}}\left[\sup _{x \in[-1,1]} I_{1}(T, x)^{\theta}\right] \leq \lim _{T \rightarrow \infty} \frac{1}{T} P_{\mathcal{Z}}\left[\log \mathcal{Z}_{\sqrt{2}}(T)\right]
$$


and

$$
\varlimsup_{T \rightarrow \infty} \frac{1}{T} \log P_{\mathcal{Z}}\left[\sup _{x \in[-1,1]} I_{2}(T, x)^{\theta}\right]=-\infty .
$$

Thus, we complete the proof.

Lemma 4.8. We have that

$$
\varlimsup_{\theta \rightarrow 0} \varlimsup_{T \rightarrow \infty} \frac{1}{\theta T} \log P_{\mathcal{Z}}\left[\sup _{x \in[-1,1]} I_{1}(T, x)^{\theta}\right] \leq \lim _{T \rightarrow \infty} \frac{1}{T} P_{\mathcal{Z}}\left[\log \mathcal{Z}_{\sqrt{2}}(T)\right]
$$

Lemma 4.9. We have that for any $\theta \in(0,1)$

$$
\varlimsup_{T \rightarrow \infty} \frac{1}{T} \log P_{\mathcal{Z}}\left[\sup _{x \in[-1,1]} I_{2}(T, x)^{\theta}\right]=-\infty .
$$

Finally, we will give the proof of (2.3) in Lemma 2.2.

Proof of (2.3). It is easy to see that for any $t>0$

$$
\begin{aligned}
P_{\mathcal{Z}}\left[\log \mathcal{Z}_{\beta}(t)\right] & =P_{\mathcal{Z}}\left[\log \int_{\mathbb{R}} \frac{\mathcal{Z}_{\beta}(t, x)}{\rho_{t}(x)} \rho_{t}(x) d x\right] \\
& \geq \int_{\mathbb{R}} P_{\mathcal{Z}}\left[\log \int_{\mathbb{R}} \frac{\mathcal{Z}_{\beta}(t, x)}{\rho_{t}(x)}\right] \rho_{t}(x) d x \\
& =P_{\mathcal{Z}}\left[\log \frac{\mathcal{Z}_{\beta}(t, 0)}{\rho_{t}(0)}\right],
\end{aligned}
$$

where we have used Jensen's inequality in the second line, and used Theorem 4.1 (v) and (4.1) in the last line.

Thus, we have from Corollary 4.6 that for any $t>0$ and $\beta>0$

$$
F_{\mathcal{Z}}(\beta) \geq-\frac{\beta^{4}}{24}
$$

Hereafter, we will look at the opposite inequality. The proof is a modification of the proof of Lemma 2.5.

It is easy to see that for $a^{\prime}(T) \in[0, \infty)$

$$
\begin{aligned}
P_{\mathcal{Z}}\left[\mathcal{Z}_{\beta}(T)^{\theta}\right] & \leq \sum_{k=-a^{\prime}(T)}^{a^{\prime}(T)} P_{\mathcal{Z}}\left[\left(\int_{2 k-1}^{2 k+1} \mathcal{Z}_{\beta}(T, x) d x\right)^{\theta}\right] \\
& +P_{\mathcal{Z}}\left[\int_{-\infty}^{-a^{\prime}(T)} \mathcal{Z}_{\beta}(T, x) d x\right]^{\theta}+P_{\mathcal{Z}}\left[\int_{a^{\prime}(T)}^{\infty} \mathcal{Z}_{\beta}(T, x) d x\right]^{\theta}
\end{aligned}
$$

If $\lim _{T \rightarrow \infty} \frac{a^{\prime}(T)}{T^{3}}>0$, then for any $\theta \in(0,1)$

$$
\varlimsup_{T \rightarrow \infty} \frac{1}{T} \log \left(P_{\mathcal{Z}}\left[\int_{-\infty}^{-a^{\prime}(T)} \mathcal{Z}_{\beta}(T, x) d x\right]^{\theta}+P_{\mathcal{Z}}\left[\int_{a^{\prime}(T)}^{\infty} \mathcal{Z}_{\beta}(T, x) d x\right]^{\theta}\right)=-\infty
$$

We denote

$$
\exp \left(A_{\beta}(T, x)\right)=\frac{\mathcal{Z}_{\beta}(T, x)}{\rho_{T}(x)}, \quad T>0, \quad x \in \mathbb{R}
$$


We remark that for fixed $T>0$, the distribution of $A_{\beta}(T, x)$ is stationary in $x \in \mathbb{R}$ by Theorem $4.1(\mathrm{v})$. Thus, we find that

$$
\begin{aligned}
P_{\mathcal{Z}}\left[\left(\int_{2 k-1}^{2 k+1} \mathcal{Z}_{\beta}(T, x) d x\right)^{\theta}\right] & =P_{\mathcal{Z}}\left[\left(\int_{-1}^{1} \exp \left(A_{\beta}(T, x+2 k)\right) \rho_{T}(x+2 k) d x\right)^{\theta}\right] \\
& \leq P_{\mathcal{Z}}\left[\sup _{x \in[-1,1]} \exp \left(\theta A_{\beta}(T, x)\right)\right]\left(\int_{-1}^{1} \rho_{T}(x+2 k) d x\right)^{\theta} .
\end{aligned}
$$

Since

$$
\sum_{k=-\infty}^{\infty}\left(\int_{-1}^{1} \rho_{T}(x+2 k) d x\right)^{\theta}<\infty
$$

it is enough to show that

$$
\varlimsup_{\theta \rightarrow 0} \varlimsup_{T \rightarrow \infty} \frac{1}{T \theta} \log P_{\mathcal{Z}}\left[\sup _{x \in[-1,1]} \exp \left(\theta A_{\beta}(T, x)\right)\right] \leq-\frac{\beta^{4}}{24}
$$

When we consider the time reversal, it is enough to show that

$$
\varlimsup_{\theta \rightarrow 0} \varlimsup_{T \rightarrow \infty} \frac{1}{T \theta} \log P_{\mathcal{Z}}\left[\sup _{x \in[-1,1]} \mathcal{Z}_{\beta}^{x}(T, 0)^{\theta}\right] \leq-\frac{\beta^{4}}{24}
$$

We know

$$
\begin{aligned}
\mathcal{Z}_{\sqrt{2}}^{x}(T, 0) & =\int_{A(T)} \mathcal{Z}_{\beta}^{x}(1, w) \int_{\mathbb{R}} \mathcal{Z}_{\beta}(1, w ; T, 0) d w \\
& +\int_{A(T)^{c}} \mathcal{Z}_{\beta}^{x}(1, w) \int_{\mathbb{R}} \mathcal{Z}_{\beta}(1, w ; T, 0) d w \\
& =I_{1}^{\prime}(T, x)+I_{2}^{\prime}(T, x)
\end{aligned}
$$

in a similar manner to the proof of Lemma 2.5. Then, we find that

$$
P_{\mathcal{Z}}\left[\left(\mathcal{Z}_{\beta}^{x}(T, x)\right)^{\theta}\right] \leq P_{\mathcal{Z}}\left[\sup _{x \in[-1,1]}\left|I_{1}^{\prime}(T, x)\right|^{\theta}\right]+P_{\mathcal{Z}}\left[\sup _{x \in[-1,1]}\left|I_{2}^{\prime}(T, x)\right|^{\theta}\right]
$$

and we obtain by using the same argument as the proof of Lemma 2.5 (we will omit the proof) that

$$
\varlimsup_{\theta \rightarrow 0} \varlimsup_{T \rightarrow \infty} \frac{1}{T \theta} \log P_{\mathcal{Z}}\left[\sup _{x \in[-1,1]}\left|I_{1}^{\prime}(T, x)\right|^{\theta}\right] \leq \varlimsup_{T \rightarrow \infty} \frac{1}{T} P_{\mathcal{Z}}\left[\log \mathcal{Z}_{\beta}(T, 0)\right]=-\frac{\beta^{4}}{24}
$$

and

$$
\varlimsup_{\theta \rightarrow 0} \varlimsup_{T \rightarrow \infty} \frac{1}{T \theta} \log P_{\mathcal{Z}}\left[\sup _{x \in[-1,1]}\left|I_{2}^{\prime}(T, x)\right|^{\theta}\right]=-\infty
$$




\subsection{Proof of Lemma 4.9}

It is easy to see from Theorem 4.1 (i) that

$$
\varlimsup_{T \rightarrow \infty} \frac{1}{T} \log P_{\mathcal{Z}}\left[I_{2}(T, 0)^{\theta}\right] \leq \varlimsup_{T \rightarrow \infty} \frac{\theta}{T} \log \int_{A(T)^{c}} \rho_{1}(0, w) d w=-\infty
$$

Thus, it is enough to show that

$$
\varlimsup_{T \rightarrow \infty} \frac{1}{T} \log P_{\mathcal{Z}}\left[\sup _{x, y \in[-1,1]}\left|I_{2}(T, x)^{\theta}-I_{2}(T, y)^{\theta}\right|\right]=-\infty
$$

Applying (3.7) to the continuous function $I_{2}(T, x)^{\theta}$ with $d=1, x=0$, Hölder's inequality yields that

$$
\begin{aligned}
& P_{\mathcal{Z}}\left[\sup _{y \in[-1,1]}\left|I_{2}(T, y)^{\theta}-I_{2}(T, 0)^{\theta}\right|\right] \\
& \leq C_{p, q}\left(\int_{-1}^{1} \int_{-1}^{1} \frac{P_{\mathcal{Z}}\left[\left|I_{2}(T, u)-I_{2}(T, v)\right|^{\theta p}\right]}{|u-v|^{p q}} d u d v\right)^{\frac{1}{p}} .
\end{aligned}
$$

for some $p>1, q>0$ with $p q>2$.

We will show that for $\theta \in(0,1)$, there exist $p \geq 1$ and $q>0$ with $p q>2$ and $p \theta>1$ such that

$$
\varlimsup_{T \rightarrow \infty} \frac{1}{T} \log \left(\int_{-1}^{1} \int_{-1}^{1} \frac{P_{\mathcal{Z}}\left[\left|I_{2}(T, u)-I_{2}(T, v)\right|^{\theta p}\right]}{|u-v|^{p q}} d u d v\right)^{\frac{1}{p}}=-\infty
$$

Proof of Lemma 4.9. We have from the definition that

$$
\begin{aligned}
I_{2}(T, u)-I_{2}(T, v) & =\int_{2 T+1}^{\infty}\left(\mathcal{Z}_{\sqrt{2}}^{u}(1, w)-\mathcal{Z}_{\sqrt{2}}^{v}(1, w)\right) \mathcal{Z}_{\sqrt{2}}^{(1, w)}(T) d w \\
& +\int_{-\infty}^{-2 T-1}\left(\mathcal{Z}_{\sqrt{2}}^{u}(1, w)-\mathcal{Z}_{\sqrt{2}}^{v}(1, w)\right) \mathcal{Z}_{\sqrt{2}}^{(1, w)}(T) d w \\
& =: J_{1}^{T}(u, v)+J_{2}^{T}(u, v)
\end{aligned}
$$

and therefore it is enough to show that for $\theta \in(0,1)$, there exist $p \geq 1$ and $q>0$ with $p q>2$ such that

$$
\varlimsup_{T \rightarrow \infty} \frac{1}{T} \log \left(\int_{-1}^{1} \int_{-1}^{1} \frac{P_{\mathcal{Z}}\left[\left|J_{1}^{T}(u, v)\right|^{\theta p}\right]}{|u-v|^{p q}} d u d v\right)^{\frac{1}{p}}=-\infty
$$


We have from Hölder's inequality that for $\theta p>1$

$$
\begin{aligned}
& P_{\mathcal{Z}}\left[\left|J_{1}^{T}(u, v)\right|^{\theta p}\right]=P_{\mathcal{Z}}\left[\left|\int_{2 T+1}^{\infty}\left(\mathcal{Z}_{\sqrt{2}}^{u}(1, w)-\mathcal{Z}_{\sqrt{2}}^{v}(1, w)\right) \mathcal{Z}_{\sqrt{2}}^{(1, w)}(T) d w\right|^{\theta p}\right] \\
& \leq P_{\mathcal{Z}}\left[\int_{2 T+1}^{\infty}\left|\frac{\mathcal{Z}_{\sqrt{2}}^{u}(1, w)-\mathcal{Z}_{\sqrt{2}}^{v}(1, w)}{\rho_{1}(2, w)}\right|^{\theta p} \mathcal{Z}_{\sqrt{2}}^{(1, w)}(T)^{\theta p} \rho_{1}(2, w) d w\right] \\
&\left.\times\left(\int_{2 T+1}^{\infty} \rho_{1}(2, w) d w\right)^{\theta p-1}\right] \\
&=\int_{2 T+1}^{\infty} P_{\mathcal{Z}}\left[\left|\frac{\mathcal{Z}_{\sqrt{2}}^{u}(1, w)-\mathcal{Z}_{\sqrt{2}}^{v}(1, w)}{\rho_{1}(2, w)}\right|^{\theta p}\right] \rho_{1}(2, w) d w \\
& \times P_{\mathcal{Z}}\left[\mathcal{Z}_{\sqrt{2}}(T-1)^{\theta p}\right]\left(\int_{2 T+1}^{\infty} \rho_{1}(2, w) d w\right)^{\theta p-1}
\end{aligned}
$$

where we have used Theorem 4.1 (ii) and (vi) in the last equality.

Since we have that for any $\theta p>1$

$$
\varlimsup_{T \rightarrow \infty} \frac{1}{T} \log \left(\int_{2 T+1}^{\infty} \rho_{1}(2, w) d w\right)^{\theta p-1}=-\infty,
$$

we obtain (4.4) from the following propositions.

Proposition 4.10. For any $r \geq 2$, we have that

$$
\varlimsup_{T \rightarrow \infty} \frac{1}{T} \log P_{\mathcal{Z}}\left[\mathcal{Z}_{\sqrt{2}}(T-1)^{r}\right]<\infty
$$

Proposition 4.11. Let $\theta \in(0,1)$. For $p=\frac{10}{\theta}>1$ and $q=\frac{3 \theta}{10}$,

$$
\sup _{T \geq 1} \int_{2 T+1}^{\infty} \int_{-1}^{1} \int_{-1}^{1} P_{\mathcal{Z}}\left[\frac{\left|\mathcal{Z}_{\sqrt{2}}^{u}(1, w)-\mathcal{Z}_{\sqrt{2}}^{v}(1, w)\right|^{\theta p}}{|u-v|^{p q}}\right] \rho_{1}(2, w)^{1-\theta p} d u d v d w<\infty
$$

The proofs of these propositions are postponed to section 5 .

\subsection{Proof of Lemma 4.8}

We recall the definition of $I_{1}(T, x)$ :

$$
\begin{aligned}
I_{1}(T, x) & =\int_{-2 T-1}^{2 T+1} \mathcal{Z}_{\sqrt{2}}^{x}(1, w) \mathcal{Z}_{\sqrt{2}}^{(1, w)}(T) d w \\
& =\int_{-2 T-1}^{2 T+1} \frac{\mathcal{Z}_{\sqrt{2}}^{x}(1, w)}{\rho_{1}(x, w)} \mathcal{Z}_{\sqrt{2}}^{(1, w)}(T) \rho_{1}(x, w) d w .
\end{aligned}
$$

Proof of Lemma 4.8. We have

$$
\begin{aligned}
\left|I_{1}(T, x)\right|^{\theta} & \leq \sum_{k=-T}^{T}\left(\int_{2 k-1}^{2 k+1} \mathcal{Z}_{\sqrt{2}}^{x}(1, w) \mathcal{Z}_{\sqrt{2}}^{(1, w)}(T) d w\right)^{\theta} \\
& :=\sum_{k=-T}^{T} I_{1}^{(k)}(T, x)^{\theta}
\end{aligned}
$$


where we have used $(x+y)^{\theta} \leq x^{\theta}+y^{\theta}$ for $x, y \geq 0$.

It is clear that

$$
\begin{aligned}
\sup _{x \in[-1,1]}\left|I_{1}^{(k)}(T, x)\right|^{\theta} \leq & \sup _{x \in[-1,1], w \in[2 k-1,2 k+1]}\left|\frac{\mathcal{Z}_{\sqrt{2}}^{x}(1, w)}{\rho_{L}(n(k), w)}\right|^{\theta} \\
& \times\left(\int_{\mathbb{R}} \rho_{L}(n(k), u) \mathcal{Z}_{\sqrt{2}}^{(1, u)}(T) d u\right)^{\theta},
\end{aligned}
$$

where $L \in \mathbb{N}$ is taken large later and $n(k)=\left\{\begin{array}{ll}1, & k \geq 1 \\ 0, & k=0 \\ -1, & k \leq-1\end{array}\right.$.

Thus, we have that

$$
\begin{aligned}
P_{\mathcal{Z}}\left[\sup _{x \in[-1,1]}\left|I_{1}(T, x)\right|^{\theta}\right] & \leq \sum_{k=-T}^{T} P_{\mathcal{Z}}\left[\sup _{x \in[-1,1], w \in[2 k-1,2 k+1]}\left|\frac{\mathcal{Z}_{\sqrt{2}}^{x}(1, w)}{\rho_{L}(n(k), w)}\right|^{\theta}\right] \\
& \times P_{\mathcal{Z}}\left[\left(\int_{\mathbb{R}} \rho_{L}(n(k), u) \mathcal{Z}_{\sqrt{2}}^{(1, u)}(T) d u\right)^{\theta}\right]
\end{aligned}
$$

We will prove the following proposition:

Proposition 4.12. There exist $L$ and a constant $C_{9}>0$ such that for $k \in \mathbb{Z}$

$$
P_{\mathcal{Z}}\left[\sup _{x \in[-1,1], w \in[2 k-1,2 k+1]}\left|\frac{\mathcal{Z}_{\sqrt{2}}^{x}(1, w)}{\rho_{L}(n(k), w)}\right|\right] \leq C_{9}
$$

Then, we have

$$
\begin{aligned}
& P_{\mathcal{Z}}\left[\sup _{x \in[-1,1]}\left|I_{1}(T, x)\right|^{\theta}\right] \\
& \leq C_{9}^{\theta} \sum_{k=-T}^{T} P_{\mathcal{Z}}\left[\left(\int_{\mathbb{R}} \rho_{L}(n(k), u) \mathcal{Z}_{\sqrt{2}}^{(1, u)}(T) d u\right)^{\theta}\right]
\end{aligned}
$$

and therefore

$$
\begin{aligned}
& \varlimsup_{\theta \rightarrow 0} \varlimsup_{T \rightarrow \infty} \frac{1}{T \theta} \log P_{\mathcal{Z}}\left[\sup _{x \in[-1,1]}\left|I_{1}(T, x)\right|^{\theta}\right] \\
& \leq \varlimsup_{\theta \rightarrow 0} \varlimsup_{T \rightarrow \infty} \frac{1}{T \theta} \log \sum_{k=-T}^{T} P_{\mathcal{Z}}\left[\left(\int_{\mathbb{R}} \rho_{L}(n(k), u) \mathcal{Z}_{\sqrt{2}}^{(1, u)}(T) d u\right)^{\theta}\right] .
\end{aligned}
$$


Also, we can obtain that

$$
\begin{aligned}
& P_{\mathcal{Z}}\left[\left(\int_{\mathbb{R}} \rho_{L}(n(k), u) \mathcal{Z}_{\sqrt{2}}^{(1, u)}(T) d u\right)^{\theta}\right] \\
& =P_{\mathcal{Z}}\left[\left(\mathcal{Z}_{\sqrt{2}}^{n(k)}(T+L) \frac{1}{\int_{\mathbb{R}} \frac{\mathcal{Z}_{\sqrt{2}}^{n(k)}(L, u)}{\rho_{L}(n(k), u)} \nu^{(n(k), L)}(u) d u}\right]\right. \\
& \leq P_{\mathcal{Z}}\left[\left(\mathcal{Z}_{\sqrt{2}}^{n(k)}(T+L)\right)^{\frac{\theta}{1-\theta}}\right]^{1-\theta} P_{\mathcal{Z}}\left[\frac{1}{\int_{\mathbb{R}} \frac{\mathcal{Z}_{\sqrt{2}}^{n(k)}(L, u)}{\rho_{L}(n(k), u)} \nu^{(n(k), L)}(u) d u}\right]^{\theta} \\
& \leq P_{\mathcal{Z}}\left[\left(\mathcal{Z}_{\sqrt{2}}^{n(k)}(T+L)\right)^{\frac{\theta}{1-\theta}}\right]^{1-\theta} P_{\mathcal{Z}}\left[\frac{\rho_{L}(n(k), 0)}{\mathcal{Z}_{\sqrt{2}}^{n(k)}(L, 0)}\right]^{\theta} \\
& \leq C_{L} P_{\mathcal{Z}}\left[\left(\mathcal{Z}_{\sqrt{2}}^{n(k)}(T+L)\right)^{\frac{\theta}{1-\theta}}\right]^{1-\theta}
\end{aligned}
$$

where $\nu^{(n(k), L)}(u)$ is the probability density function on $\mathbb{R}$ given by

$$
\nu^{(n(k), L)}(u)=\frac{1}{\int_{\mathbb{R}} \rho_{L}(n(k), u) \mathcal{Z}_{\sqrt{2}}^{(L, u)}(T+L) d u} \rho_{L}(n(k), u) \mathcal{Z}_{\sqrt{2}}^{(L, u)}(L+T),
$$

we have used Theorem $4.1(\mathrm{v})$ in the second inequality, and $C_{L}$ is a constant comes from Corollary 4.3. Thus, we have from Lemma 4.4 that

$$
\begin{aligned}
& \varlimsup_{\theta \rightarrow 0} \varlimsup_{T \rightarrow \infty} \frac{1}{T \theta} \log P_{\mathcal{Z}}\left[\left(\int_{\mathbb{R}} \rho_{L}(u-1) \mathcal{Z}_{\sqrt{2}}^{(1, u)}(T) d u\right)^{\theta}\right] \\
& \leq \mathcal{F}_{\mathcal{Z}}(\sqrt{2})
\end{aligned}
$$

and we can complete the proof of Lemma 2.5.

\section{Proof of propositions}

In this section, we give a proof of propositions given in section 4 .

Throughout this section, we often use the following hypercontractivity of Wiener chaos, where we omit definition of some notations and change the statement of Theorem to adjust our purpose:

Theorem 5.1. [26, Theorem 5.10] Let $X \in L^{2}(\Omega, \mathcal{F}, P)$ with Gaussian Hilbert space $H$. Then, $X$ has the following Wiener chaos decomposition

$$
X=\sum_{n=0}^{\infty} X_{n}, \quad X_{n} \in H^{: n}
$$

Then, we have that for any $r \geq 2$ and for $n \geq 0$

$$
P\left[\left|X_{n}\right|^{r}\right]^{\frac{1}{r}} \leq(r-1)^{\frac{n}{2}} P\left[\left|X_{n}\right|^{2}\right]^{\frac{1}{2}} .
$$


In addition, if we assume that $X \in L^{r}(\Omega, \mathcal{F}, P)$ for some $2 \leq r<\infty$, then we have that

$$
P\left[|X|^{r}\right]^{\frac{1}{r}} \leq\left(\sum_{n \geq 0}(r-1)^{n} P\left[\left|X_{n}\right|^{2}\right]\right)^{\frac{1}{2}}
$$

Also, we will usually use the equations and the inequalities in appendix.

\subsection{Proof of Proposition 4.10}

Proof of Proposition 4.10. $\mathcal{Z}_{\sqrt{2}}(T-1)$ has the following Wiener chaos decomposition:

$$
\begin{aligned}
& \mathcal{Z}_{\sqrt{2}}(T-1) \\
& =1+\sum_{n \geq 1} 2^{n / 2} \int_{\Delta_{n}(0, T-1)} \int_{\mathbb{R}^{n}}\left(\int_{\mathbb{R}} \boldsymbol{\rho}_{n}(\mathbf{t}, \mathbf{x} \mid 0, x ; T, y) d y\right) \mathcal{W}\left(d t_{1} d x_{1}\right) \cdots \mathcal{W}\left(d t_{n} d x_{n}\right) \\
& =: \sum_{n=0}^{\infty} K^{(n)}(T-1) .
\end{aligned}
$$

Then, we have from (A.9) that

$$
P_{\mathcal{Z}}\left[K^{(n)}(T-1)^{2}\right]=\frac{(T-1)^{n / 2}}{\Gamma\left(\frac{n+1}{2}\right)}
$$

and hence, hypercontractivity of Wiener chaos (Theorem 5.1) yields that for $r \geq 2$

$$
\begin{aligned}
P_{\mathcal{Z}}\left[\left|K^{(n)}(T-1)\right|^{r}\right]^{1 / r} & \leq(r-1)^{n / 2} P_{\mathcal{Z}}\left[K^{(n)}(T-1)^{2}\right]^{1 / 2} \\
& =\frac{(r-1)^{n / 2}(T-1)^{n / 4}}{\Gamma\left(\frac{n+1}{2}\right)^{1 / 2}}
\end{aligned}
$$

Thus, we have that for $r \geq 2$

$$
P_{\mathcal{Z}}\left[\mathcal{Z}_{\sqrt{2}}(T-1)^{r}\right] \leq\left(\sum_{n \geq 0} \frac{(r-1)^{n / 2}(T-1)^{n / 4}}{\Gamma\left(\frac{n+1}{2}\right)^{1 / 2}}\right)^{r}
$$

It is easy to see from (3.16) that

$$
\varlimsup_{t \rightarrow \infty} \frac{1}{T} \log P_{\mathcal{Z}}\left[\mathcal{Z}_{\sqrt{2}}(T-1)^{r}\right]<\infty
$$

Remark 5.2. In a similar way, we can prove that for any $r \geq 2, \beta \in \mathbb{R}, x \in \mathbb{R}$ and $T>0$, $\mathcal{Z}_{\beta}(T), \mathcal{Z}_{\beta}(T, x) \in L^{r}$. Thus, we may applies the latter part in Theorem 5.1 to any linear combinations of $\mathcal{Z}_{\beta}(T)$ and $\mathcal{Z}_{\beta}(T, x)$.

\subsection{Proof of Proposition 4.11}

The proof of Proposition 4.11 is almost the same as the one of Proposition 4.10.

We remark that $\mathcal{Z}_{\sqrt{2}}^{u}(1, w)-\mathcal{Z}_{\sqrt{2}}^{v}(1, w)$ has the following Wiener chaos expansion:

$$
\begin{aligned}
& \mathcal{Z}_{\sqrt{2}}^{u}(1, w)-\mathcal{Z}_{\sqrt{2}}^{v}(1, w) \\
& =\rho_{1}(u, w)-\rho_{1}(v, w) \\
& +\sum_{n \geq 1} 2^{n / 2} \int_{\Delta_{n}(0,1)} \int_{\mathbb{R}^{n}}\left(\boldsymbol{\rho}_{n}(\mathbf{t}, \mathbf{x} \mid 0, u ; 1, w)-\boldsymbol{\rho}_{n}(\mathbf{t}, \mathbf{x} \mid 0, v ; 1, w)\right) \mathcal{W}\left(d t_{1} d x_{1}\right) \cdots \mathcal{W}\left(d t_{n} d x_{n}\right) \\
& =: \sum_{n=0}^{\infty} L^{(n)}(u, v ; w) .
\end{aligned}
$$


Then, we have that for $u, v \in[-1,1]$

$$
\begin{array}{ll}
P_{\mathcal{Z}}\left[L^{(n)}(u, v ; w)^{2}\right] \\
= \begin{cases}\left(\rho_{1}(u, w)-\rho_{1}(v, w)\right)^{2}, & n=0 \\
2^{n} \int_{\Delta_{n}(0,1)} \int_{\mathbb{R}^{n}}\left(\boldsymbol{\rho}_{n}(\mathbf{t}, \mathbf{x} \mid 0, u ; 1, w)-\boldsymbol{\rho}_{n}(\mathbf{t}, \mathbf{x} \mid 0, v ; 1, w)\right)^{2} d \mathbf{t} d \mathbf{x}, & n \geq 1\end{cases}
\end{array}
$$

We will prove that there exists a polynomial $H$ with degree 4 such that for any $n \geq 0$ and for $u, v \in[-1,1], w \geq 2 T+1$

$$
P_{\mathcal{Z}}\left[L^{(n)}(u, v ; w)^{2}\right] \leq \frac{|u-v||H(w)| \rho_{1}(2, w)^{2}}{\Gamma\left(\frac{n}{2} \vee 1\right)}
$$

If (5.2) holds, then Proposition 4.11 follows. Indeed, hypercontractivity of Wiener chaos (Theorem 5.1) yields that

$$
P_{\mathcal{Z}}\left[\left|\sum_{n=0}^{\infty} L^{(n)}(u, v ; w)\right|^{r}\right] \leq\left(\sum_{n=0}^{\infty}(r-1)^{n / 2} P_{\mathcal{Z}}\left[\left|L^{(n)}(u, v ; w)\right|^{2}\right]\right)^{\frac{r}{2}}
$$

Then, we have that

$$
\begin{aligned}
& P_{\mathcal{Z}}\left[\left|\mathcal{Z}_{\sqrt{2}}^{u}(1, w)-\mathcal{Z}_{\sqrt{2}}^{v}(1, w)\right|^{r}\right] \\
& \leq|u-v|^{\frac{r}{2}}|H(w)|^{r / 2} \rho_{1}(2, w)^{r}\left(\sum_{n \geq 0} \frac{(r-1)^{n}}{\Gamma\left(\frac{n}{2} \vee 1\right)}\right)^{r / 2} .
\end{aligned}
$$

Thus, taking $r=p \theta=10$ and $p q=3$, we have that

$$
\begin{aligned}
& \int_{2 T+1}^{\infty} \int_{-1}^{1} \int_{-1}^{1} P_{\mathcal{Z}}\left[\frac{\left|\mathcal{Z}_{\sqrt{2}}^{u}(1, w)-\mathcal{Z}_{\sqrt{2}}^{v}(1, w)\right|^{10}}{|u-v|^{3}}\right] \rho_{1}(2, w)^{1-10} d u d v d w \\
& \leq C_{10} \int_{T}^{\infty}|H(w)|^{5} \rho_{1}(2, w) d w
\end{aligned}
$$

(5.2) for $n=0$

(A.3), (A.13), (A.14), and (A.15) yield that

$$
\begin{aligned}
L^{(0)}(u, v ; w)^{2}= & \rho_{2}(0) \rho_{\frac{1}{2}}(u, w)+\rho_{2}(0) \rho_{\frac{1}{2}}(v, w)-2 \rho_{2}(u, v) \rho_{\frac{1}{2}}\left(\frac{u+v}{2}, w\right) \\
\leq & \frac{|u-v|^{2}}{8 \sqrt{\pi}}\left(\rho_{\frac{1}{2}}(u, w)+\rho_{\frac{1}{2}}(v, w)\right) \\
& \quad+\rho_{2}(u, v)\left(\rho_{\frac{1}{2}}(u, w)+\rho_{\frac{1}{2}}(v, w)-2 \rho_{\frac{1}{2}}\left(\frac{u+v}{2}, w\right)\right) \\
\leq & \frac{|u-v|^{2}}{4 \sqrt{\pi}} \rho_{\frac{1}{2}}(2, w)+|u-v|^{2}|h(w)| \rho_{\frac{1}{2}}(2, w) \\
\leq & |u-v|\left|H_{1}(w)\right| \rho_{1}(2, w)^{2}
\end{aligned}
$$

for $u, v \in[-1,1]$ and $w \geq 2 T+1$, where $H_{1}(w)$ is a polynomial with degree 4 .

(5.2) for $n \geq 1$ 
We have that for $n \geq 1$

$$
\begin{aligned}
& P_{\mathcal{Z}}\left[L^{(n)}(u, v ; w)^{2}\right] \\
& =2^{n} \int_{0}^{1} d t_{0} \int_{\mathbb{R}} d x\left(\rho_{t_{0}}(u, x)-\rho_{t_{0}}(v, x)\right)^{2}\left(\int_{\Delta_{n-1}\left(t_{0}, 1\right)} \int_{\mathbb{R}^{n-1}} \boldsymbol{\rho}_{n-1}\left(\mathbf{t}, \mathbf{x} \mid t_{0}, x ; 1, w\right)^{2} d \mathbf{t} d \mathbf{x}\right) \\
& \stackrel{(A .8)}{=} \int_{0}^{1} d t_{0} \int_{\mathbb{R}} d x\left(\rho_{t_{0}}(u, x)-\rho_{t_{0}}(v, x)\right)^{2} \frac{\left(1-t_{0}\right)^{\frac{n-2}{2}}}{\Gamma\left(\frac{n}{2}\right)} \rho_{\frac{1-t_{0}}{2}}(x, w) \\
& \stackrel{(A .13)}{=} \int_{0}^{1} d t_{0} \int_{\mathbb{R}} d x\left(\rho_{\frac{t_{0}}{2}}(u, x)+\rho_{\frac{t_{0}}{2}}(v, x)\right)\left(\rho_{2 t_{0}}(0)-\rho_{2 t_{0}}\left(\frac{u-v}{2}\right)\right) \frac{\left(1-t_{0}\right)^{\frac{n-2}{2}} \rho_{\frac{1-t_{0}}{2}}(x, w)}{\Gamma\left(\frac{n}{2}\right)} \\
& +\int_{0}^{1} d t_{0} \int_{\mathbb{R}} d x \rho_{2 t_{0}}(u, v)\left(\rho_{\frac{t_{0}}{2}}(u, x)+\rho_{\frac{t_{0}}{2}}(v, x)-2 \rho_{\frac{t_{0}}{2}}\left(\frac{u+v}{2}, x\right)\right) \frac{\left(1-t_{0}\right)^{\frac{n-2}{2}} \rho_{\frac{1-t_{0}}{2}}(x, w)}{\Gamma\left(\frac{n}{2}\right)} \\
& \stackrel{(A .4)}{=}\left(\rho_{\frac{1}{2}}(u, w)+\rho_{\frac{1}{2}}(v, w)\right) \int_{0}^{1}\left(\rho_{2 t_{0}}(0)-\rho_{2 t_{0}}\left(\frac{u-v}{2}\right)\right) \frac{\left(1-t_{0}\right)^{\frac{n-2}{2}}}{\Gamma\left(\frac{n}{2}\right)} d t_{0} \\
& +\left(\rho_{\frac{1}{2}}(u, w)+\rho_{\frac{1}{2}}(v, w)-2 \rho_{\frac{1}{2}}\left(\frac{u+v}{2}, w\right)\right) \int_{0}^{1} \frac{\rho_{2 t_{0}}(u, v)\left(1-t_{0}\right)^{\frac{n-2}{2}}}{\Gamma\left(\frac{n}{2}\right)} d t_{0} \\
& \stackrel{(A .14)}{\leq} 2 \rho_{\frac{1}{2}}(2, w) \int_{0}^{1}\left(\rho_{2 t_{0}}(0)-\rho_{2 t_{0}}\left(\frac{u-v}{2}\right)\right) \frac{\left(1-t_{0}\right)^{\frac{n-2}{2}}}{\Gamma\left(\frac{n}{2}\right)} d t_{0} \\
& +\frac{|u-v|^{2}}{4} G_{u, v, w, w}\left(\frac{u-v}{2}\right) \int_{0}^{1} \frac{t_{0}^{-\frac{1}{2}}\left(1-t_{0}\right)^{\frac{n-2}{2}}}{2 \sqrt{\pi} \Gamma\left(\frac{n}{2}\right)} d t_{0}
\end{aligned}
$$

Thus, we have from (A.1), (A.12), and (A.15) that for $n=1$

$$
P_{\mathcal{Z}}\left[L^{(n)}(u, v ; w)^{2}\right] \leq|u-v|\left|H_{2}(w)\right| \rho_{1}(2, w)^{2}
$$

and from (A.1), (A.10), and (A.15) that for $n \geq 2$

$$
\begin{aligned}
& P_{\mathcal{Z}}\left[L^{(n)}(u, v ; w)^{2}\right] \\
& \leq \frac{2 \rho_{\frac{1}{2}}(2, w)}{\Gamma\left(\frac{n}{2}\right)} \int_{0}^{1}\left(\rho_{2 t_{0}}(0)-\rho_{2 t_{0}}\left(\frac{u-v}{2}\right)\right) d t_{0}+\frac{\sqrt{\pi} \rho_{\frac{1}{2}}(2, w)}{\Gamma\left(\frac{n+1}{2}\right)}|u-v|^{2} G_{u, v, w, w}\left(\frac{u-v}{2}\right) \\
& \leq \frac{|u-v|}{\Gamma\left(\frac{n}{2}\right)}\left|H_{3}(w)\right| \rho_{1}(2, w)^{2}
\end{aligned}
$$

for $u, v \in[-1,1]$ and $w \geq 2 T+1$, where $H_{2}(w)$ and $H_{3}(w)$ are polynomials with degree 4 .

\subsection{Proof of Proposition 4.12}

Proof. We will see only the case $k \geq 1$. The case $k \leq-1$ follows by symmetry and the case $k=0$ is proved by modification.

We consider a function on $[-1,1] \times[1, \infty)$

$$
f(x, w)=\frac{\mathcal{Z}_{\sqrt{2}}^{x}(1, w)}{\rho_{L}(1, w)} .
$$

Then, we have from Lemma 4.1 (i) that

$$
P_{\mathcal{Z}}[f(x, w)]=\frac{\rho_{1}(x, w)}{\rho_{L}(1, w)} \leq C_{L} .
$$


From (3.8), it is enough to show that for some $p \geq 1$ and $q>0$ with $p q>4$, there exist $\eta_{p}>p q-2$ and $C_{11}>0$ such that

$$
P_{\mathcal{Z}}\left[\left|f(x, w)-f\left(x^{\prime}, w^{\prime}\right)\right|^{p}\right] \leq C_{11}\left|(x, w)-\left(x^{\prime}, w^{\prime}\right)\right|^{\eta_{p}},
$$

where we should remark that $C_{11}$ is a constant independent of $x, x^{\prime} \in[-1,1], w, w^{\prime} \in$ $[2 k-1,2 k+1]$, and $k \geq 1$.

Indeed,

$$
\begin{aligned}
& P_{\mathcal{Z}}\left[\left|\sup _{x \in[-1,1], w \in[2 k-1,2 k+1]}\right| f(x, w)||\right] \\
& \leq P_{\mathcal{Z}}[|f(0,2 k)|]+P_{\mathcal{Z}}\left[\left|\sup _{x, y \in[-1,1], w, w^{\prime} \in[2 k-1,2 k+1]}\right| f(x, w)-f\left(y, w^{\prime}\right) \mid\right] \\
& \leq C_{L}+C_{p, q, 2} 2^{-\frac{4}{p}}\left(\int_{B_{2}((0,2 k))} \int_{B_{2}((0,2 k))} P_{\mathcal{Z}}\left[\frac{\left|f(x, w)-f\left(y, w^{\prime}\right)\right|^{p}}{\left|(x, w)-\left(y, w^{\prime}\right)\right|^{p q}}\right] d(x, w) d\left(y, w^{\prime}\right)\right)^{\frac{1}{p}} \\
& \leq C_{L}+C_{p, q, 2} 2^{-\frac{4}{p}} C_{12}\left(\int_{B_{2}((0,2 k))} \int_{B_{2}((0,2 k))}\left|(x, w)-\left(y, w^{\prime}\right)\right|^{\eta_{p}-p q} d(x, w) d\left(y, w^{\prime}\right)\right)^{\frac{1}{p}} \\
& \leq C_{12},
\end{aligned}
$$

where $C_{12}$ is a constant independent of $k \geq 1$, and therefore Proposition 4.12 follows.

If $w, w^{\prime} \in[2 k-1,2 k+1]$, then we have

$$
\begin{aligned}
\left|f(x, w)-f\left(x^{\prime}, w^{\prime}\right)\right| & \leq\left|\frac{\mathcal{Z}_{\sqrt{2}}^{x}(1, w)-\mathcal{Z}_{\sqrt{2}}^{x^{\prime}}\left(1, w^{\prime}\right)}{\rho_{L}(1, w)}\right|+\mathcal{Z}_{\sqrt{2}}^{x^{\prime}}\left(1, w^{\prime}\right)\left|\frac{\rho_{L}(1, w)-\rho_{L}\left(1, w^{\prime}\right)}{\rho_{L}(1, w) \rho_{L}\left(1, w^{\prime}\right)}\right| \\
& \leq\left|\frac{\mathcal{Z}_{\sqrt{2}}^{x}(1, w)-\mathcal{Z}_{\sqrt{2}}^{x^{\prime}}\left(1, w^{\prime}\right)}{\rho_{L}(1, w)}\right|+\frac{1}{L} \frac{\left|w-w^{\prime}\right|\left|w+w^{\prime}-2\right|}{\rho_{L}\left(1, w \vee w^{\prime}\right)} \mathcal{Z}_{\sqrt{2}}^{x^{\prime}}\left(1, w^{\prime}\right)
\end{aligned}
$$

for $x, x^{\prime} \in[-1,1]$.

To prove (5.3), we will show that for every $p>2$, there exist $C_{p, 1}>0$ and $C_{p, 2}>0$ such that

$$
P_{\mathcal{Z}}\left[\left|\mathcal{Z}_{\sqrt{2}}^{x}(1, w)-\mathcal{Z}_{\sqrt{2}}^{x^{\prime}}\left(1, w^{\prime}\right)\right|^{p}\right] \leq C_{p, 1}\left|(x, w)-\left(x^{\prime}, w^{\prime}\right)\right|^{p / 2} \rho_{1}\left(2, w \wedge w^{\prime}\right)^{p}
$$

and

$$
P_{\mathcal{Z}}\left[\left|\frac{\mathcal{Z}_{\sqrt{2}}^{x^{\prime}}\left(1, w^{\prime}\right)}{\rho_{L}\left(1, w \vee w^{\prime}\right)}\right|^{p}\right] \leq C_{p, 2}
$$

for $x, x^{\prime} \in[-1,1], w, w^{\prime} \in[2 k-1,2 k+1]$, and $k \geq 1$. Then, (5.3) follows for some $L=L(p) \geq 2$ large enough.

$\mathcal{Z}_{\sqrt{2}}^{x}(1, w)$ has the Wiener chaos expansion

$$
\begin{aligned}
\mathcal{Z}_{\sqrt{2}}^{x}(1, w) & =\rho_{1}(w-x)+\sum_{n \geq 1} 2^{\frac{n}{2}} \int_{\Delta_{n}(1)} \boldsymbol{\rho}_{n}(\mathbf{t}, \mathbf{x} \mid 0, x ; 1, w) W\left(t_{1}, x_{1}\right) \cdots W\left(t_{n}, x_{n}\right) \\
& =\sum_{n \geq 0} 2^{\frac{n}{2}} M^{(n)}(x, w) .
\end{aligned}
$$

Then, we have from (A.8) that

$$
P_{\mathcal{Z}}\left[\left(M^{(n)}(x, w)\right)^{2}\right]=\frac{1}{2^{n+1} \Gamma\left(\frac{n+1}{2}\right)} \rho_{\frac{1}{2}}(x, w),
$$


and Theorem 5.1 implies that for $p \geq 2$

$$
\begin{aligned}
P_{\mathcal{Z}}\left[\mathcal{Z}_{\sqrt{2}}^{x^{\prime}}\left(1, w^{\prime}\right)^{p}\right] & \leq\left(\sum_{n \geq 0}(p-1)^{n} 2^{n}\left(\frac{1}{2^{n+1} \Gamma\left(\frac{n+1}{2}\right)} \rho_{\frac{1}{2}}\left(x^{\prime}, w^{\prime}\right)\right)\right)^{\frac{p}{2}} \\
& \leq C_{p, 3} \rho_{1}\left(x^{\prime}, w^{\prime}\right)^{p} .
\end{aligned}
$$

Thus, (5.5) holds for $L$ large enough.

For (5.4), we can estimate from (A.13) and (A.15) that

$$
\begin{aligned}
& \left|M^{(0)}(x, w)-M^{(0)}\left(x^{\prime}, w^{\prime}\right)\right|^{2} \\
& \stackrel{(A .14)}{\leq} \frac{\left|\left(w-w^{\prime}\right)-\left(x-x^{\prime}\right)\right|^{2}}{8 \sqrt{\pi}}\left(\rho_{\frac{1}{2}}(x, w)+\rho_{\frac{1}{2}}\left(x^{\prime}, w^{\prime}\right)\right) \\
& +\rho_{2}\left(x-x^{\prime}, w-w^{\prime}\right)\left|\frac{\left(w-w^{\prime}\right)-\left(x-x^{\prime}\right)}{2}\right|^{2} G_{x, x^{\prime}, w, w^{\prime}}\left(\frac{w-x-w^{\prime}+x^{\prime}}{2}\right) \\
& \leq\left|w-w^{\prime}-\left(x-x^{\prime}\right)\right|\left|H_{4}\left(w+w^{\prime}\right)\right| \rho_{\frac{1}{2}}\left(2, w \wedge w^{\prime}\right),
\end{aligned}
$$

where $H_{4}$ is a polynomial with degree 4 and also

$$
\begin{aligned}
& P_{\mathcal{Z}}\left[\left|M^{(1)}(x, w)-M^{(1)}\left(x^{\prime}, w^{\prime}\right)\right|^{2}\right] \\
& =\int_{0}^{1} \int_{\mathbb{R}}\left(\rho_{s}(x, y) \rho_{1-s}(y, w)-\rho_{s}\left(x^{\prime}, y\right) \rho_{1-s}\left(y, w^{\prime}\right)\right)^{2} d s d y \\
& \stackrel{(A .1),(A .3)}{=} \int_{0}^{1} \int_{\mathbb{R}}\left(\rho_{2 s}(0) \rho_{2(1-s)}(0)\left(\rho_{\frac{s}{2}}(x, y) \rho_{\frac{1-s}{2}}(y, w)+\rho_{\frac{s}{2}}\left(x^{\prime}, y\right) \rho_{\frac{1-s}{2}}\left(y, w^{\prime}\right)\right)\right. \\
& \left.\quad-2 \rho_{2 s}\left(x, x^{\prime}\right) \rho_{2(1-s)}\left(w, w^{\prime}\right) \rho_{\frac{s}{2}}\left(\frac{x+x^{\prime}}{2}, y\right) \rho_{\frac{1-s}{2}}\left(y, \frac{w+w^{\prime}}{2}\right)\right) d s d y \\
& \stackrel{(A .4)}{=} \int_{0}^{1}\left(\rho_{2 s}(0) \rho_{2(1-s)}(0)\left(\rho_{\frac{1}{2}}(x, w)+\rho_{\frac{1}{2}}\left(x^{\prime}, w^{\prime}\right)\right)\right. \\
& =\left(\rho_{\frac{1}{2}}(x, w)+\rho_{\frac{1}{2}}\left(x^{\prime}, w^{\prime}\right)\right) \int_{0}^{1} d s \rho_{2 s}(0)\left(\rho_{2(1-s)}(0)-\rho_{2(1-s)}\left(w, w^{\prime}\right)\right) \\
& +\left(\rho_{\frac{1}{2}}(x, w)+\rho_{\frac{1}{2}}\left(x^{\prime}, w^{\prime}\right)\right) \int_{0}^{1} d s\left(\rho_{2 s}(0)-\rho_{2 s}\left(x, x^{\prime}\right)\right) \rho_{2(1-s)}\left(w, w^{\prime}\right) \\
& +\left(\rho_{\frac{1}{2}}(x, w)+\rho_{\frac{1}{2}}\left(x^{\prime}, w^{\prime}\right)-2 \rho_{\frac{1}{2}}\left(\frac{x+x^{\prime}}{2}, \frac{w+w^{\prime}}{2}\right)\right) \int_{0}^{1} d s \rho_{2 s}\left(x, x^{\prime}\right) \rho_{2(1-s)}\left(w, w^{\prime}\right) \\
& \leq\left(\rho_{\frac{1}{2}}(x, w)+\rho_{\frac{1}{2}}\left(x^{\prime}, w^{\prime}\right)\right) \int_{0}^{1}\left(2 \rho_{2 s}(0)-\rho_{2 s}\left(x, x^{\prime}\right)-\rho_{2 s}\left(w, w^{\prime}\right)\right) \rho_{2-2 s}(0) d s \\
& +\left|\frac{w-x-w^{\prime}+x^{\prime}}{2}\right|^{2} G_{x, x^{\prime}, w, w^{\prime}}\left(\frac{w-x-w^{\prime}+x^{\prime}}{2}\right) \int_{0}^{1} d s \rho_{2 s}\left(x, x^{\prime}\right) \rho_{2(1-s)}\left(w, w^{\prime}\right) \\
& +(A .12),(A .15) \\
& \leq \\
& \leq \\
& \mid
\end{aligned}
$$

for $x, x^{\prime} \in[-1,1]$ and $w, w^{\prime} \in[2 k-1,2 k+1](k \geq 1)$, where $H_{5}$ is a polynomial with degree 4 . 
Also, we have that for $n \geq 2$

$$
\begin{aligned}
P_{\mathcal{Z}}\left[\left|M^{(n)}(x, w)-M^{(n)}\left(x^{\prime}, w^{\prime}\right)\right|^{2}\right] & \\
=\int_{\Delta_{n}(0,1)} \int_{\mathbb{R}^{n}}\left(\rho_{t_{1}}\left(x, x_{1}\right) \rho_{1-t_{n}}\left(x_{n}, w\right)-\right. & \left.\rho_{t_{1}}\left(x^{\prime}, x_{1}\right) \rho_{1-t_{n}}\left(x_{n}, w^{\prime}\right)\right)^{2} \\
& \times \boldsymbol{\rho}_{n-2}\left(\mathbf{t}^{\prime}, \mathbf{x}^{\prime} \mid t_{1}, x_{1} ; t_{n}, x_{n}\right)^{2} d \mathbf{t} d \mathbf{x},
\end{aligned}
$$

where $\mathbf{t}^{\prime}=\left(t_{2}, \cdots, t_{n-1}\right), \mathbf{x}^{\prime}=\left(x_{2}, \cdots, x_{n-1}\right)$ for $\mathbf{t}=\left(t_{1}, \cdots, t_{n}\right)$ and $\mathbf{x}=\left(x_{1}, \cdots, x_{n}\right)$.

Thus, we have from (A.1), (A.3), and (A.8) that

$$
\begin{aligned}
& P_{\mathcal{Z}}\left[\left|M^{(n)}(x, w)-M^{(n)}\left(x^{\prime}, w^{\prime}\right)\right|^{2}\right] \\
& =\int_{0}^{1} d t_{1} \int_{t_{1}}^{1} d t_{n} \int_{\mathbb{R}^{2}} d x_{1} d x_{n} \frac{\left(t_{n}-t_{1}\right)^{\frac{n-3}{2}} \rho_{\frac{t_{n}-t_{1}}{2}}\left(x_{1}, x_{n}\right)}{2^{n-1} \Gamma\left(\frac{n-1}{2}\right)} \\
& \times\left(\rho_{2 t_{1}}(0) \rho_{2\left(1-t_{n}\right)}(0) \rho_{\frac{t_{1}}{2}}\left(x, x_{1}\right) \rho_{\frac{1-t_{n}}{2}}\left(x_{n}, w\right)+\rho_{2 t_{1}}(0) \rho_{2\left(1-t_{n}\right)}(0) \rho_{\frac{t_{1}}{2}}\left(x^{\prime}, x_{1}\right) \rho_{\frac{1-t_{n}}{2}}\left(x_{n}, w^{\prime}\right)\right. \\
& \left.\quad-2 \rho_{2 t_{1}}\left(x, x^{\prime}\right) \rho_{\frac{t_{1}}{2}}\left(\frac{x+x^{\prime}}{2}, x_{1}\right) \rho_{2\left(1-t_{n}\right)}\left(w, w^{\prime}\right) \rho_{\frac{1-t_{n}}{2}}\left(x_{n}, \frac{w+w^{\prime}}{2}\right)\right) \\
& =\int_{0}^{1} d s \int_{s}^{1} d t \frac{(t-s)^{\frac{n-3}{2}}}{2^{n-1} \Gamma\left(\frac{n-1}{2}\right)} \\
& \quad \times\left(\rho_{2 s}(0) \rho_{2(1-t)}(0) \rho_{\frac{1}{2}}(x, w)\right. \\
& \quad+\rho_{2 s}(0) \rho_{2(1-t)}(0) \rho_{\frac{1}{2}}\left(x^{\prime}, w^{\prime}\right) \\
& =: \int_{0}^{1} \int_{s}^{1} \frac{(t-s)^{\frac{n-3}{2}}}{2^{n-1} \Gamma\left(\frac{n-1}{2}\right)}\left(N_{s, t}^{1}\left(x, x^{\prime}\right) \rho_{2(1-t)}\left(w, w^{\prime}\right) \rho_{\frac{1}{2}}\left(\frac{\left.\left.x+x^{\prime}\right)+N_{s, t}^{2}\left(x, x^{\prime} w, w^{\prime}\right)+N_{s, t}^{3}\left(x, x^{\prime} w, w^{\prime}\right)\right) d t d s}{2}\right)\right)
\end{aligned}
$$

where we set

$$
\begin{aligned}
& N_{s, t}^{1}\left(x, x^{\prime} w, w^{\prime}\right)=\rho_{2 s}(0) \rho_{2(1-t)}(0)\left(\rho_{\frac{1}{2}}(x, w)+\rho_{\frac{1}{2}}\left(x^{\prime}, w^{\prime}\right)-2 \rho_{\frac{1}{2}}\left(\frac{x+x^{\prime}}{2}, \frac{w+w^{\prime}}{2}\right)\right) \\
& N_{s, t}^{2}\left(x, x^{\prime} w, w^{\prime}\right)=2 \rho_{\frac{1}{2}}\left(\frac{x+x^{\prime}}{2}, \frac{w+w^{\prime}}{2}\right) \rho_{2 s}(0)\left(\rho_{2(1-t)}(0)-\rho_{2(1-t)}\left(w, w^{\prime}\right)\right) \\
& N_{s, t}^{3}\left(x, x^{\prime} w, w^{\prime}\right)=2 \rho_{\frac{1}{2}}\left(\frac{x+x^{\prime}}{2}, \frac{w+w^{\prime}}{2}\right) \rho_{2(1-t)}\left(w, w^{\prime}\right)\left(\rho_{2 s}(0)-\rho_{2 s}\left(x, x^{\prime}\right)\right) .
\end{aligned}
$$

Thus, we have that

$$
\begin{aligned}
& \int_{0}^{1} \int_{s}^{1} \frac{(t-s)^{\frac{n-3}{2}}}{2^{n-1} \Gamma\left(\frac{n-1}{2}\right)} N_{s, t}^{1}\left(x, x^{\prime} w, w^{\prime}\right) d t d s \\
& =\frac{1}{2^{n+1} \Gamma\left(\frac{n+1}{2}\right)}\left(\rho_{\frac{1}{2}}(x, w)+\rho_{\frac{1}{2}}\left(x^{\prime}, w^{\prime}\right)-2 \rho_{\frac{1}{2}}\left(\frac{x+x^{\prime}}{2}, \frac{w+w^{\prime}}{2}\right)\right) \\
& =\frac{1}{2^{n+1} \Gamma\left(\frac{n+1}{2}\right)}\left|\frac{w-x-w^{\prime}+x^{\prime}}{2}\right|^{2} G_{x, x^{\prime}, w, w^{\prime}}\left(\frac{w-x-w^{\prime}+x^{\prime}}{2}\right) \\
& \int_{0}^{1} \int_{s}^{1} \frac{(t-s)^{\frac{n-3}{2}}}{2^{n-1} \Gamma\left(\frac{n-1}{2}\right)} N_{s, t}^{2}\left(x, x^{\prime} w, w^{\prime}\right) d t d s \\
& =\frac{2}{2^{n} \Gamma\left(\frac{n}{2}\right)} \rho_{\frac{1}{2}}\left(\frac{x+x^{\prime}}{2}, \frac{w+w^{\prime}}{2}\right) \int_{0}^{1} t^{\frac{n-2}{2}}\left(\rho_{2(1-t)}(0)-\rho_{2(1-t)}\left(w, w^{\prime}\right)\right) d t
\end{aligned}
$$


and

$$
\begin{aligned}
& \int_{0}^{1} \int_{s}^{1} \frac{(t-s)^{\frac{n-3}{2}}}{2^{n-1} \Gamma\left(\frac{n-1}{2}\right)} N_{s, t}^{3}\left(x, x^{\prime} w, w^{\prime}\right) d t d s \\
& \leq \frac{2}{2^{n} \Gamma\left(\frac{n}{2}\right)} \rho_{\frac{1}{2}}\left(\frac{x+x^{\prime}}{2}, \frac{w+w^{\prime}}{2}\right) \int_{0}^{1}(1-s)^{\frac{n-2}{2}}\left(\rho_{2 s}(0)-\rho_{2 s}\left(w, w^{\prime}\right)\right) d s .
\end{aligned}
$$

Thus, we have from (A.10) and (A.15) that

$$
P_{\mathcal{Z}}\left[\left|M^{(n)}(x, w)-M^{(n)}\left(x^{\prime}, w^{\prime}\right)\right|^{2}\right] \leq\left(\left|w-w^{\prime}\right|+\left|x-x^{\prime}\right|\right)\left|H_{6}\left(w+w^{\prime}\right)\right| \rho_{\frac{1}{2}}\left(2, w \wedge w^{\prime}\right)
$$

for for $x, x^{\prime} \in[-1,1]$ and $w, w^{\prime} \in[2 k-1,2 k+1](k \geq 1)$, where $H_{6}$ is a polynomial with degree 4 .

We should remark that $H_{4}, H_{5}$, and $H_{6}$ can be chosen independent of $k \geq 1$.

Then, hypercontractivity of Wiener chaos (Theorem 5.1) implies that

$$
\begin{aligned}
& P_{\mathcal{Z}}\left[\left|\mathcal{Z}_{\sqrt{2}}^{x}(1, w)-\mathcal{Z}_{\sqrt{2}}^{x^{\prime}}\left(1, w^{\prime}\right)\right|^{p}\right] \\
& \leq\left|\left(w-w^{\prime}\right)-\left(x-x^{\prime}\right)\right|^{p / 2}\left(\sum_{k \geq 0}(p-1)^{n}\left(\frac{H_{7}\left(w+w^{\prime}\right)}{2^{n-1} \Gamma\left(\frac{n-1}{2} \vee 1\right)} \rho_{\frac{1}{2}}\left(2, w \wedge w^{\prime}\right)\right)\right)^{\frac{p}{2}} \\
& \leq C_{p}\left(\left|w-w^{\prime}\right|+\left|x-x^{\prime}\right|\right)^{p / 4} \rho_{1}\left(2, w \wedge w^{\prime}\right)^{p}
\end{aligned}
$$

for $x, x^{\prime} \in[-1,1], w, w^{\prime} \in[2 k-1,2 k+1]$, and for $k \geq 1$, so (5.4) follows.

\section{A Heat kernel}

\section{A.1 Some formulas of heat kernel}

Here, we give some formulas on heat kernels for calculations in this paper. We set

$$
\rho_{t}(x-y)=\rho_{t}(x, y)=\frac{1}{\sqrt{2 \pi t}} \exp \left(-\frac{(y-x)^{2}}{2 t}\right)
$$

for $x, y \in \mathbb{R}$ and $t>0$ and

$$
\begin{aligned}
& \boldsymbol{\rho}_{0}(\mathbf{t}, \mathbf{x} \mid s, x ; t, y)=\rho_{t-s}(x, y) \\
& \boldsymbol{\rho}_{1}(\mathbf{t}, \mathbf{x} \mid s, x ; t, y)=\rho_{t_{1}-s}\left(x, x_{1}\right) \rho_{t-t_{1}}\left(x_{1}, y\right) \\
& \boldsymbol{\rho}_{n}(\mathbf{t}, \mathbf{x} \mid s, x ; t, y)=\rho_{t_{1}-s}\left(x, x_{1}\right)\left(\prod_{i=1}^{n-1} \rho_{t_{i+1}-t_{i}}\left(x_{i}, x_{i+1}\right)\right) \rho_{t-t_{n}}\left(x_{n}, y\right)
\end{aligned}
$$

for $x, y \in \mathbb{R}, \mathbf{x}=\left(x_{1}, \cdots, x_{n}\right) \in \mathbb{R}^{n}, 0 \leq s<t$ and for

$$
\mathbf{t} \in \Delta_{n}(s, t)=\left\{\mathbf{t}=\left(t_{1}, \cdots, t_{n}\right): s<t_{1}<\cdots<t_{n} \leq t\right\}
$$

where we may regard $\mathbf{t}=s, \mathbf{x}=x$ for $n=0$. Then, we have that for $w, x, y, z \in \mathbb{R}$ and $s, t \in[0, \infty)$

$$
\rho_{t}(x)^{2}=\frac{1}{2 \sqrt{\pi t}} \rho_{\frac{t}{2}}(x)=\rho_{2 t}(0) \rho_{\frac{t}{2}}(x)
$$

and

$$
\rho_{t}(x, w) \rho_{t}(y, z)=\rho_{2 t}(x-y, w-z) \rho_{\frac{t}{2}}\left(\frac{x+y}{2}, \frac{w+z}{2}\right) .
$$


In particular,

$$
\rho_{t}(x, w) \rho_{t}(y, w)=\rho_{2 t}(x, y) \rho_{\frac{t}{2}}\left(\frac{x+y}{2}, w\right)
$$

and

$$
\int_{\mathbb{R}} \rho_{s}(x, y) \rho_{t}(y, z) d y=\rho_{t+s}(x, z) .
$$

Therefore, we have that

$$
\begin{aligned}
\int_{\mathbb{R}}\left(\rho_{t}(x, w)-\rho_{t}(y, w)\right)^{2} d w & =2\left(\rho_{2 t}(0)-\rho_{2 t}(x, y)\right) \\
\int_{\mathbb{R}^{n}} \boldsymbol{\rho}_{n}\left(\mathbf{t}, \mathbf{x} \mid t_{0}, x ; t_{n+1}, y\right)^{2} d \mathbf{x} & =\frac{1}{2^{n+1} \pi^{\frac{n+1}{2}}} \rho_{\frac{t_{n+1}-t_{0}}{2}}(x, y) \prod_{i=0}^{n} \frac{1}{\sqrt{t_{i+1}-t_{i}}},
\end{aligned}
$$

and

$$
\int_{\mathbb{R}^{n}}\left(\int_{\mathbb{R}} \boldsymbol{\rho}_{n}\left(\mathbf{t}, \mathbf{x} \mid t_{0}, x ; t_{n+1}, y\right) d y\right)^{2} d \mathbf{x}=\frac{1}{2^{n} \pi^{\frac{n}{2}}} \prod_{i=0}^{n-1} \frac{1}{\sqrt{t_{i+1}-t_{i}}},
$$

for $n \geq 1, x, y \in \mathbb{R}, 0 \leq t_{0}<t_{n+1}, \mathbf{t}=\left(t_{1}, \cdots, t_{k}\right) \in \Delta_{n}\left(t_{0}, t_{n+1}\right)$.

Also, since for $0<a<b$ and for $\alpha, \beta \in(0, \infty)$,

$$
\int_{a}^{b}(x-a)^{\alpha-1}(b-x)^{\beta-1} d x=(b-a)^{\alpha+\beta-1} B(\alpha, \beta)=(b-a)^{\alpha+\beta-1} \frac{\Gamma(\alpha) \Gamma(\beta)}{\Gamma(\alpha+\beta)},
$$

we have

$$
\begin{aligned}
\int_{\Delta_{n}\left(t_{0}, t_{n+1}\right)} \int_{\mathbb{R}^{n}} \boldsymbol{\rho}_{n}\left(\mathbf{t}, \mathbf{x} \mid t_{0}, x ; t_{n+1}, y\right)^{2} d \mathbf{t} d \mathbf{x} & =\frac{\left(t_{n+1}-t_{0}\right)^{\frac{n-1}{2}}}{2^{n+1} \Gamma\left(\frac{n+1}{2}\right)} \rho_{\frac{t_{n+1}-t_{0}}{2}}(x, y), \\
\int_{\Delta_{n}\left(t_{0}, t_{n+1}\right)} \int_{\mathbb{R}^{n}}\left(\int_{\mathbb{R}} \boldsymbol{\rho}_{n}\left(\mathbf{t}, \mathbf{x} \mid t_{0}, x ; t_{n+1}, y\right) d y\right)^{2} d \mathbf{t} d \mathbf{x} & =\frac{\left(t_{n+1}-t_{0}\right)^{\frac{n}{2}}}{2^{n} \Gamma\left(\frac{n+2}{2}\right)} .
\end{aligned}
$$

\section{A.2 Inequalities on heat equation}

We have the following inequalities:

$$
\begin{aligned}
\int_{0}^{t}\left(\rho_{s}(0)-\rho_{s}(x)\right) d s & =\frac{|x|}{2 \sqrt{\pi}} \int_{\frac{\left.|x|\right|^{2}}{2 t}}^{\infty} \frac{1}{u^{\frac{3}{2}}}(1-\exp (-u)) d u \\
& \leq \frac{|x|}{2 \sqrt{\pi}} \int_{0}^{\infty} u^{-\frac{3}{2}}(1 \wedge u) d u=\frac{2|x|}{\sqrt{\pi}} \\
\int_{0}^{t} \rho_{s}(x) d s & \leq \int_{0}^{t} \rho_{s}(0) d s=\sqrt{\frac{2 t}{\pi}}
\end{aligned}
$$

for any $t>0$ and $x \in \mathbb{R}$ and therefore it follows that

$$
\begin{aligned}
& \int_{0}^{t}\left(\rho_{s}(0)-\rho_{s}(x)\right)(t-s)^{-\frac{1}{2}} d s \\
& =\int_{0}^{\frac{t}{2}}\left(\rho_{s}(0)-\rho_{s}(x)\right)(t-s)^{-\frac{1}{2}} d s+\int_{\frac{t}{2}}^{t}\left(\rho_{s}(0)-\rho_{s}(x)\right)(t-s)^{-\frac{1}{2}} d s \\
& \leq\left(\frac{t}{2}\right)^{-\frac{1}{2}} \int_{0}^{t}\left(\rho_{s}(0)-\rho_{s}(x)\right) d s+\frac{x^{2}}{\sqrt{\pi} t^{3 / 2}} \int_{\frac{t}{2}}^{t}(t-s)^{\frac{1}{2}} d s \\
& \leq 2 \sqrt{\frac{2}{\pi}} \frac{|x|}{\sqrt{t}}+\sqrt{\frac{2}{\pi}} \frac{|x|^{2}}{t} .
\end{aligned}
$$


Also, we have

$$
\begin{aligned}
& \left(\rho_{t}(x, w)-\rho_{t}\left(y, w^{\prime}\right)\right)^{2} \\
& =\left(\rho_{2 t}(0)-\rho_{2 t}\left(x-y, w-w^{\prime}\right)\right)\left(\rho_{\frac{t}{2}}(x, w)+\rho_{\frac{t}{2}}\left(y, w^{\prime}\right)\right) \\
& \quad+\rho_{2 t}\left(x-y, w-w^{\prime}\right)\left(\rho_{\frac{t}{2}}(x, w)+\rho_{\frac{t}{2}}\left(y, w^{\prime}\right)-2 \rho_{\frac{t}{2}}\left(\frac{x+y}{2}, \frac{w+w^{\prime}}{2}\right)\right) \\
& \leq \frac{\left|\left(w-w^{\prime}\right)-(x-y)\right|^{2}}{8 \sqrt{\pi t^{3}}}\left(\rho_{\frac{t}{2}}(x, w)+\rho_{\frac{t}{2}}\left(y, w^{\prime}\right)\right) \\
& \quad+\rho_{2 t}\left(x-y, w-w^{\prime}\right)\left(\rho_{\frac{t}{2}}(x, w)+\rho_{\frac{t}{2}}\left(y, w^{\prime}\right)-2 \rho_{\frac{t}{2}}\left(\frac{x+y}{2}, \frac{w+w^{\prime}}{2}\right)\right)
\end{aligned}
$$

for $t>0$ and $w, w^{\prime}, x, y \in \mathbb{R}$.

Throughout this paper, we need to estimate (A.13) only for $t=1, x, y \in[-1,1]$ and $\left|w-w^{\prime}\right| \leq 2$. Applying Taylor's theorem to $f_{x, y, w, w^{\prime}}(u)=\rho_{\frac{1}{2}}\left(u, \frac{w+w^{\prime}-x-y}{2}\right)$, there exist $\theta_{1}$ and $\theta_{2} \in(0,1)$ such that

$$
\begin{aligned}
& f_{x, y, w, w^{\prime}}(u)+f_{x, y, w, w^{\prime}}(-u)-2 f_{x, y, w, w^{\prime}}(0) \\
& =f_{x, y, w, w^{\prime}}^{\prime \prime}(0) u^{2}+\frac{1}{6}\left(f_{x, y, w, w^{\prime}}^{(3)}\left(\theta_{1} u\right)-f_{x, y, w, w^{\prime}}^{(3)}\left(-\theta_{2} u\right)\right) u^{3} \\
& =u^{2}\left(\left(-2+\left(w+w^{\prime}-x-y\right)^{2}\right) f_{x, y, w, w^{\prime}}(0)\right. \\
& \quad+u\left(2\left(\theta_{1} u-\frac{w+w^{\prime}-x-y}{2}\right)-\frac{4\left(\theta_{1} u-\frac{w+w^{\prime}-x-y}{2}\right)^{3}}{3}\right) f_{x, y, w, w^{\prime}}\left(\theta_{1} u\right) \\
& \left.\quad-u\left(2\left(-\theta_{2} u-\frac{w+w^{\prime}-x-y}{2}\right)-\frac{4\left(-\theta_{2} u-\frac{w+w^{\prime}-x-y}{2}\right)^{3}}{3}\right) f_{x, y, w, w^{\prime}}\left(-\theta_{2} u\right)\right) \\
& =: u^{2} G_{x, y, w, w^{\prime}}(u) .
\end{aligned}
$$

We remark that there exists a polynomial $h$ with degree 4 such that

$$
\begin{aligned}
& \left|G_{x, y, w, w^{\prime}}\left(\frac{(w-x)-\left(w^{\prime}-y\right)}{2}\right)\right| \\
& \leq\left|h\left(\frac{w+w^{\prime}}{2}\right)\right| \rho_{\frac{1}{2}}\left(|w-2| \wedge|w+2| \wedge\left|w^{\prime}-2\right| \wedge\left|w^{\prime}+2\right|\right),
\end{aligned}
$$

for $x, y \in[-1,1]$, and for $w, w^{\prime} \in \mathbb{R}$ with $\left|w-w^{\prime}\right| \leq 2$.

\section{References}

[1] Tom Alberts, Konstantin Khanin, and Jeremy Quastel. The continuum directed random polymer. J. Stat. Phys., Vol. 154, No. 1-2, pp. 305-326, 2014. MR-3162542

[2] Tom Alberts, Konstantin Khanin, and Jeremy Quastel. The intermediate disorder regime for directed polymers in dimension $1+1$. Ann. Probab., Vol. 42, No. 3, pp. 1212-1256, 2014. MR-3189070

[3] Kenneth S. Alexander and Gökhan Yildirim. Directed polymers in a random environment with a defect line. Electron. J. Probab., Vol. 20, pp. no. 6, 1-20, 2015. MR-3311219

[4] Gideon Amir, Ivan Corwin, and Jeremy Quastel. Probability distribution of the free energy of the continuum directed random polymer in $1+1$ dimensions. Comm. Pure Appl. Math., Vol. 64, No. 4, pp. 466-537, 2011. MR-2796514 
[5] Emil Artin. The gamma function. Translated by Michael Butler. Athena Series: Selected Topics in Mathematics. Holt, Rinehart and Winston, New York-Toronto-London, 1964. MR-0165148

[6] Quentin Berger and Hubert Lacoin. The high-temperature behavior for the directed polymer in dimension 1+2. http://arxiv.org/abs/1506.09055, To appear in Annales de l'Institut Henri Poincar'e., 2015. MR-3606747

[7] Quentin Berger and Fabio Lucio Toninelli. On the critical point of the random walk pinning model in dimension $d=3$. Electron. J. Probab., Vol. 15, No. 21, pp. 654-683, 2010. MR2650777

[8] Lorenzo Bertini and Giambattista Giacomin. Stochastic Burgers and KPZ equations from particle systems. Comm. Math. Phys., Vol. 183, No. 3, pp. 571-607, 1997. MR-1462228

[9] Matthias Birkner. A condition for weak disorder for directed polymers in random environment. Electron. Comm. Probab., Vol. 9, pp. 22-25 (electronic), 2004. MR-2041302

[10] Matthias Birkner, Andreas Greven, and Frank den Hollander. Collision local time of transient random walks and intermediate phases in interacting stochastic systems. Electron. J. Probab., Vol. 16, No. 20, pp. 552-586, 2011. MR-2786642

[11] Matthias Birkner and Rongfeng Sun. Annealed vs quenched critical points for a random walk pinning model. Ann. Inst. Henri Poincaré Probab. Stat., Vol. 46, No. 2, pp. 414-441, 2010. MR-2667704

[12] Matthias Birkner and Rongfeng Sun. Disorder relevance for the random walk pinning model in dimension 3. Ann. Inst. Henri Poincaré Probab. Stat., Vol. 47, No. 1, pp. 259-293, 2011. MR-2779405

[13] Erwin Bolthausen. A note on the diffusion of directed polymers in a random environment. Comm. Math. Phys., Vol. 123, No. 4, pp. 529-534, 1989. MR-1006293

[14] Francesco Caravenna, Fabio Lucio Toninelli, and Niccolo Torri. Universality for the pinning model in the weak coupling regime. arXiv preprint arXiv:1505.04927, 2015. MR-3693960

[15] Philippe Carmona and Yueyun Hu. On the partition function of a directed polymer in a Gaussian random environment. Probab. Theory Related Fields, Vol. 124, No. 3, pp. 431-457, 2002. MR-1939654

[16] Philippe Carmona and Yueyun Hu. Strong disorder implies strong localization for directed polymers in a random environment. ALEA Lat. Am. J. Probab. Math. Stat., Vol. 2, pp. 217-229, 2006. MR-2249669

[17] Francis Comets. Directed polymers in random environment. Saint Flour lecture notes, 2016. MR-3444835

[18] Francis Comets and Vu-Lan Nguyen. Localization in log-gamma polymers with boundaries. Probab. Theory Related Fields, Vol. 166, No. 1-2, pp. 429-461, 2016. MR-3547743

[19] Francis Comets, Tokuzo Shiga, and Nobuo Yoshida. Directed polymers in a random environment: path localization and strong disorder. Bernoulli, Vol. 9, No. 4, pp. 705-723, 2003. MR-1996276

[20] Francis Comets, Tokuzo Shiga, and Nobuo Yoshida. Probabilistic analysis of directed polymers in a random environment: a review. In Stochastic analysis on large scale interacting systems, Vol. 39 of Adv. Stud. Pure Math., pp. 115-142. Math. Soc. Japan, Tokyo, 2004. MR-2073332

[21] Francis Comets and Vincent Vargas. Majorizing multiplicative cascades for directed polymers in random media. ALEA Lat. Am. J. Probab. Math. Stat., Vol. 2, pp. 267-277, 2006. MR2249671

[22] Francis Comets and Nobuo Yoshida. Directed polymers in random environment are diffusive at weak disorder. Ann. Probab., Vol. 34, No. 5, pp. 1746-1770, 2006. MR-2271480

[23] Nicos Georgiou and Timo Seppäläinen. Large deviation rate functions for the partition function in a log-gamma distributed random potential. Ann. Probab., Vol. 41, No. 6, pp. 4248-4286, 2013. MR-3161474

[24] Giambattista Giacomin, Hubert Lacoin, and Fabio Lucio Toninelli. Disorder relevance at marginality and critical point shift. Vol. 47, No. 1, pp. 148-175, 2011. MR-2779401 
Free energy of $1+1$ DPRE

[25] David A Huse and Christopher L Henley. Pinning and roughening of domain walls in ising systems due to random impurities. Physical review letters, Vol. 54, No. 25, pp. 2708-2711, 1985.

[26] Svante Janson. Gaussian Hilbert spaces, Vol. 129 of Cambridge Tracts in Mathematics. Cambridge University Press, Cambridge, 1997. MR-1474726

[27] Ioannis Karatzas and Steven E. Shreve. Brownian motion and stochastic calculus, Vol. 113 of Graduate Texts in Mathematics. Springer-Verlag, New York, second edition, 1991. MR-1121940

[28] Hubert Lacoin. New bounds for the free energy of directed polymers in dimension $1+1$ and $1+$ 2. Comm. Math. Phys., Vol. 294, No. 2, pp. 471-503, 2010. MR-2579463

[29] Gregory F. Lawler and Vlada Limic. Random walk: a modern introduction, Vol. 123 of Cambridge Studies in Advanced Mathematics. Cambridge University Press, Cambridge, 2010. MR-2677157

[30] Michel Ledoux. The concentration of measure phenomenon, Vol. 89 of Mathematical Surveys and Monographs. American Mathematical Society, Providence, RI, 2001. MR-1849347

[31] Quansheng Liu and Frédérique Watbled. Exponential inequalities for martingales and asymptotic properties of the free energy of directed polymers in a random environment. Stochastic Process. Appl., Vol. 119, No. 10, pp. 3101-3132, 2009. MR-2568267

[32] Gregorio R. Moreno Flores. On the (strict) positivity of solutions of the stochastic heat equation. Ann. Probab., Vol. 42, No. 4, pp. 1635-1643, 2014. MR-3262487

[33] Elchanan Mossel, Ryan O’Donnell, and Krzysztof Oleszkiewicz. Noise stability of functions with low influences: invariance and optimality. Ann. of Math. (2), Vol. 171, No. 1, pp. 295-341, 2010. MR-2630040

[34] Makoto Nakashima. A remark on the bound for the free energy of directed polymers in random environment in $1+2$ dimension. J. Math. Phys., Vol. 55, No. 9, pp. 093304, 14, 2014. MR-3390799

[35] Makoto Nakashima. The free energy of the random walk pinning model. Stochastic Process. Appl., Vol. 128, No. 2, pp. 373-403, 2018. MR-3739501

[36] David Nualart. The Malliavin calculus and related topics. Probability and its Applications (New York). Springer-Verlag, Berlin, second edition, 2006. MR-2200233

[37] Jeremy Quastel. Introduction to KPZ. In Current developments in mathematics, 2011, pp. 125-194. Int. Press, Somerville, MA, 2012. MR-3098078

[38] Tomohiro Sasamoto and Herbert Spohn. Exact height distributions for the KPZ equation with narrow wedge initial condition. Nuclear Phys. B, Vol. 834, No. 3, pp. 523-542, 2010. MR-2628936

[39] Tomohiro Sasamoto and Herbert Spohn. One-dimensional kardar-parisi-zhang equation: an exact solution and its universality. Physical review letters, Vol. 104, No. 23, p. 230602, 2010.

[40] Timo Seppäläinen. Scaling for a one-dimensional directed polymer with boundary conditions. Ann. Probab., Vol. 40, No. 1, pp. 19-73, 2012. MR-2917766

[41] Fabio Lucio Toninelli. Coarse graining, fractional moments and the critical slope of random copolymers. Electron. J. Probab., Vol. 14, No. 20, pp. 531-547, 2009. MR-2480552

[42] Frédérique Watbled. Sharp asymptotics for the free energy of $1+1$ dimensional directed polymers in an infinitely divisible environment. Electron. Commun. Probab., Vol. 17, No. 53, pp. 9, 2012. MR-2999981

Acknowledgments. The author appreciates the associated editor and the referees for their careful reading and comment. 


\section{Electronic Journal of Probability Electronic Communications in Probability}

\section{Advantages of publishing in EJP-ECP}

- Very high standards

- Free for authors, free for readers

- Quick publication (no backlog)

- Secure publication $\left(\mathrm{LOCKSS}^{1}\right)$

- Easy interface (EJMS²)

\section{Economical model of EJP-ECP}

- Non profit, sponsored by $\mathrm{IMS}^{3}, \mathrm{BS}^{4}$, ProjectEuclid ${ }^{5}$

- Purely electronic

\section{Help keep the journal free and vigorous}

- Donate to the IMS open access fund ${ }^{6}$ (click here to donate!)

- Submit your best articles to EJP-ECP

- Choose EJP-ECP over for-profit journals

\footnotetext{
${ }^{1}$ LOCKSS: Lots of Copies Keep Stuff Safe http://www. lockss.org/

${ }^{2}$ EJMS: Electronic Journal Management System http://www.vtex.lt/en/ejms.html

${ }^{3}$ IMS: Institute of Mathematical Statistics http://www.imstat.org/

${ }^{4}$ BS: Bernoulli Society http://www. bernoulli-society.org/

${ }^{5}$ Project Euclid: https://projecteuclid.org/

${ }^{6}$ IMS Open Access Fund: http://www.imstat.org/publications/open.htm
} 